\title{
Metabolomics window into the role of acute kidney injury after coronary artery bypass grafting in diabetic nephropathy progression
}

\author{
Jiayi Wang ${ }^{1}$, Wenzhe Yan ${ }^{2}$, Xiang Zhou ${ }^{1}$, Yu Liu ${ }^{1}$, Chengyuan Tang ${ }^{1}$, Youming Peng ${ }^{1}$, Hong Liu ${ }^{1}, \operatorname{Lin}$ Sun ${ }^{1}$, Li \\ Xiao ${ }^{1}$, Liyu He ${ }^{\text {Corresp. } 1}$ \\ 1 Department of Nephrology, The Second Xiangya Hospital, Central South University, Key Lab of Kidney Disease and Blood Purification in Hunan, \\ Changsha, China \\ 2 Department of Hematology, The Second Xiangya Hospital, Central South University, Changsha, China \\ Corresponding Author: Liyu He \\ Email address: heliyu1124@csu.edu.cn
}

Introduction. Metabolomics has emerged as a valuable tool to discover novel biomarkers and study the pathophysiology of diabetic nephropathy (DN). However, the effect of postoperative acute kidney injury (AKI) on diabetes mellitus (DM) to chronic DN progression has not been evaluated from the perspective of metabolomics. Methods. A group of type 2 diabetes mellitus (T2DM) inpatients, who underwent off-pump coronary artery bypass grafting (CABG), were enrolled in our study. According to whether postoperative AKI occurred, patients were grouped in either the AKI group (AKI, $n=44$ ) or the non-AKI group (NAKI, $n=44$ ). Urine samples were collected from these patients before and $24 \mathrm{~h}$ after operation. Six patients from the AKI group and six patients from the NAKI group were chosen as the pilot cohort for untargeted metabolomics analysis, with the goal of identifying postoperative AKI-related metabolites. To understand the possible role of these metabolites in the chronic development of renal injury among T2DM patients, trans-4-hydroxy-L-proline and azelaic acid were quantified by targeted metabolomics analysis among 38 NAKI patients, 38 AKI patients, 46 early DN patients (DN-micro group), and 34 overt DN patients (DN-macro group). Results. Untargeted metabolomics screened 61 statistically distinguishable metabolites in postoperative urine samples, compared with preoperative urine samples. Via Venn diagram analysis, 9 of 61 were postoperative AKIrelated metabolites, including trans-4-hydroxy-L-proline, uridine triphosphate, $p$ aminobenzoate, caffeic acid, adrenochrome, ס-valerolactam, L-norleucine, 5'-deoxy-5'(methylthio) adenosine, and azelaic acid. By targeted metabolomics analysis, the level of trans-4-hydroxy-L-proline increased gradually from the NAKI group to the AKI, DN-micro, and DN-macro groups. For azelaic acid, the highest level was found in the NAKI and DNmicro groups, followed by the DN-macro group. The AKI group exhibited the lowest level of azelaic acid. Conclusions. The detection of urinary trans-4-hydroxy-L-proline after AKI PeerJ reviewing PDF | (2020:01:45338:2:0:NEW 6 Apr 2020) 
could be treated as an early warning of chronic DN progression and might be linked to renal fibrosis. Urinary azelaic acid can be used to monitor renal function noninvasively in DM and DN patients. Our results identified markers of AKI on DM and the chronic progression of DN. In addition, the progression of DN was associated with AKI-like episodes occurring in DM. 


\section{Metabolomics window into the role of acute kidney injury after coronary artery bypass grafting in diabetic nephropathy progression}

Jiayi Wang ${ }^{1}$, Wenzhe Yan $^{2}$, Xiang Zhou ${ }^{1}$, Yu Liu ${ }^{1}$, Chengyuan Tang ${ }^{1}$, Youming Peng ${ }^{1}$, Hong

$5 \mathrm{Liu}^{1}, \mathrm{Lin} \mathrm{Sun}^{1}, \mathrm{Li} \mathrm{Xiao}^{1}, \mathrm{Liyu} \mathrm{He}^{1 *}$

$6 \quad{ }^{1}$ Department of Nephrology, The Second Xiangya Hospital, Central South University, Key Lab

7 of Kidney Disease and Blood Purification in Hunan, 139 Renmin Road, Changsha, Hunan

8 410011, People's Republic of China.

$9{ }^{2}$ Department of Hematology, The Second Xiangya Hospital, Central South University,

10 Changsha, Hunan 410011, People's Republic of China.

*Corresponding author:

13 Liyu He

14 Full mailing address: Department of Nephrology, The Second Xiangya Hospital, Central South

15 University, Key Lab of Kidney Disease and Blood Purification in Hunan, 139 Renmin Road,

16 Changsha, Hunan 410011, People's Republic of China.

17 Tel: +8673185292064

18 Fax number: +8673185295843

19 Email: heliyu1124@csu.edu.cn 


\section{Abstract}

36 Introduction. Metabolomics has emerged as a valuable tool to discover novel biomarkers and

37

38

39

40

41

42

43

44

45

46

47

48

49

50

51

52

53

54

55

56

57

58

59

60

61

62

63

64

65

66

67

68

69

study the pathophysiology of diabetic nephropathy (DN). However, the effect of postoperative acute kidney injury (AKI) on diabetes mellitus (DM) to chronic DN progression has not been evaluated from the perspective of metabolomics.

Methods. A group of type 2 diabetes mellitus (T2DM) inpatients, who underwent off-pump coronary artery bypass grafting (CABG), were enrolled in our study. According to whether postoperative AKI occurred, patients were grouped in either the AKI group $(\mathrm{AKI}, \mathrm{n}=44)$ or the non-AKI group (NAKI, $n=44$ ). Urine samples were collected from these patients before and 24 $\mathrm{h}$ after operation. Six patients from the AKI group and six patients from the NAKI group were chosen as the pilot cohort for untargeted metabolomics analysis, with the goal of identifying postoperative AKI-related metabolites. To understand the possible role of these metabolites in the chronic development of renal injury among T2DM patients, trans-4-hydroxy-L-proline and azelaic acid were quantified by targeted metabolomics analysis among 38 NAKI patients, 38 AKI patients, 46 early DN patients (DN-micro group), and 34 overt DN patients (DN-macro group).

Results. Untargeted metabolomics screened 61 statistically distinguishable metabolites in postoperative urine samples, compared with preoperative urine samples. Via Venn diagram analysis, 9 of 61 were postoperative AKI-related metabolites, including trans-4-hydroxy-Lproline, uridine triphosphate, $\mathrm{p}$-aminobenzoate, caffeic acid, adrenochrome, $\delta$-valerolactam, Lnorleucine, 5'-deoxy-5'-(methylthio) adenosine, and azelaic acid. By targeted metabolomics analysis, the level of trans-4-hydroxy-L-proline increased gradually from the NAKI group to the AKI, DN-micro, and DN-macro groups. For azelaic acid, the highest level was found in the NAKI and DN-micro groups, followed by the DN-macro group. The AKI group exhibited the lowest level of azelaic acid.

Conclusions. The detection of urinary trans-4-hydroxy-L-proline after AKI could be treated as an early warning of chronic DN progression and might be linked to renal fibrosis. Urinary azelaic acid can be used to monitor renal function noninvasively in DM and DN patients. Our results identified markers of AKI on DM and the chronic progression of DN. In addition, the progression of DN was associated with AKI-like episodes occurring in DM.

\section{Introduction}

Till 2017, about 451 million adults live with diabetes mellitus (DM) in the world. Moreover, $44.4 \%$ of the type $2 \mathrm{DM}$ (T2DM) will progress to diabetic nephropathy (DN) in three decades. DN is now the most frequent cause of end stage renal disease (ESRD) (Cho et al. 2018; Hallan et al. 2006). Microalbuminuria is a useful diagnostic marker and major risk factor for DN progression; however, it has been challenged for its limited sensitivity and specificity (Chen et al. 2017; Tabaei et al. 2001). Multiple studies have explored better biomarkers for detecting early pathophysiologic changes of DN (Colhoun \& Marcovecchio 2018). 
Metabolomics, the systems biology approach for the identification and quantification of global metabolites in biological samples (Chen et al. 2018a), has emerged as a valuable tool to discover novel biomarkers of DM and DN (Arneth et al. 2019; Darshi et al. 2016). Using metabolomics, significant changes in the serum level of leucine, dihydrosphingosine, and phytoshpingosine were noted in DM and DN patient groups, compared with the controls (Zhang et al. 2009). Metabolite profiling in participants from two large, longitudinal studies also identified five branched-chain and aromatic amino acids (isoleucine, leucine, valine, tyrosine, and phenylalanin) that are highly associated with future diabetes (Wang et al. 2011). Meanwhile, metabolomics helps to elucidate amino acid metabolism, phospholipid metabolism, and lipid metabolism, which may be the important biological mechanisms that promote DM and DN (Rossi et al. 2018; Zhang et al. 2009). Urine is a noninvasive sample source that contains all renal metabolic end products. Thus, urine metabolomics has been used for predicting the development of nephropathy in T2DM patients (Chen et al. 2018a), DN progression from microto macroalbuminuria (Pena et al. 2014), and ESRD risk of T2DM patients with microalbuminuria (Tang et al. 2019).

$\mathrm{DN}$ is rarely a static condition but rather one that evolves and changes over time during the lifespan of the individual. Acute kidney injury (AKI) could participate in and promote the dynamic progression of DN. It was reported that the yearly AKI incidence of T2DM was 198/100,000, versus 27/100,000 among nondiabetic individuals (Girman et al. 2012). In animal models, ischemic AKI was more severe in diabetic mice than in non-diabetic mice (Gao et al. 2013; Peng et al. 2015). These studies indicate that DM predisposes and sensitizes patients to AKI. However, the effect of AKI on DM to chronic DN progression has not been evaluated from the perspective of metabolomics.

AKI is one of the most important complications after coronary artery bypass grafting (CABG) procedure, especially for DM patients (Bellomo et al. 2008). Hence, we choose T2DM patients undergoing elective off-pump CAGB as our main study objects to examine AKIassociated metabolites among DM patients. Untargeted metabolomics analysis was performed on the urine samples of these patients in the discovery stage. To further explore the role of identified postoperative AKI markers in the chronic development of renal injury among T2DM patients, the discovered metabolites were quantified by targeted metabolomics analysis among DM patients with or without $\mathrm{AKI}$ after $\mathrm{CABG}, \mathrm{DN}$ patients with microalbuminuria, and DN patients with macroalbuminuria. The results suggest potential biomarkers for early detection and progression of DN, as well as associate chronic DN progression with AKI-like episodes. This helps to visualize the dynamic progress of DN via metabolomics.

\section{Materials and Methods}

\section{Definitions}

T2DM was diagnosed via WHO criteria. Normoalbuminuria was defined as urinary albumin excretion rate (UAER) $<30 \mathrm{mg}$ /day; microalbuminuria was defined as UAER $=30-300$ $\mathrm{mg} /$ day, and macroalbuminuria as UAER $>300 \mathrm{mg} /$ day. T2DM with microalbuminuria (DN- 
114 micro) was defined as early DN. T2DM with macroalbuminuria (DN-macro) was defined as

115 overt DN. Postoperative AKI was defined as an absolute increase in serum creatinine by

$116 \geq 0.3 \mathrm{mg} / \mathrm{dL}(26.5 \mu \mathrm{mol} / \mathrm{L})$ from baseline within $48 \mathrm{~h}$ or $\geq 50 \%$ within 7 days after operation. The

117 staging of AKI was evaluated according to the criteria proposed by Kidney disease: Improving

118 global outcomes (KDIGO) classifications (Kellum et al. 2013). Anemia was defined as

119 hemoglobin less than $110 \mathrm{~g} / \mathrm{L}$ in females or $120 \mathrm{~g} / \mathrm{L}$ in males. The definition of chronic kidney

120 disease (CKD) followed the National Kidney Foundation-Kidney Disease Outcomes Quality

121 Initiative (K/DOQI) guidelines, and the CKD stage was defined using patient baseline estimated

122 glomerular filtration rate (eGFR) (National Kidney 2002). eGFR was calculated using the

123 Chronic Kidney Disease Epidemiology Collaboration equation (CKD-EPI).

124

125

\section{Patient recruitment and urine collection}

T2DM patients who received the elective off-pump CABG procedure between Jan 2018 and Aug 2019 at the Second Xiangya Hospital of Central South University were screened for study participation. The inclusion criteria were as follows: (1) T2DM with normoalbuminuria; (2) Age ranged from 30 to 75 years; (3) eGFR $>60 \mathrm{~mL} / \mathrm{min} / 1.73 \mathrm{~m}^{2}$. Exclusion criteria were as follows: (1) Diagnosis with type 1 diabetes mellitus (WHO criteria); (2) Other etiology of CKD; (3) AKI prior to surgery; (4) Acute and chronic inflammation of the urinary system or other systems; (5) Patients with significant coexisting illnesses likely to affect survival or combined with other systemic diseases (including but not limited to malignancy, severe heart failure, blood system diseases, autoimmune diseases, cancer patients, and AIDS); (6) Patients with insufficient clinical data; (7) Patients underwent emergent surgeries, reoperations, and combined surgeries. In total, 88 T2DM patients were enrolled, and 8 of them received the intra-aortic balloon pump (IABP) during operation. Off-pump CABG was performed using conventional techniques, and complete revascularization was achieved in all patients. The mean duration of operation was around $4 \mathrm{~h}$. Preoperative urine samples collected on the day before operation were named A group. At about $24 \mathrm{~h}$ after operation, postoperative urine samples were collected from a Foley catheter in the ICU and named B group. According to whether AKI occurred after operation, patients were grouped in the AKI group (AKI, $n=44)$ or the non-AKI group (NAKI, $n=44)$. Every patient in each group supplied both pre-and postoperative urine samples; thus, the AKI group was further subdivided into the AKI-A group and AKI-B group. The NAKI group was subdivided into the NAKI-A group and NAKI-B group. There were no deaths or dialysis needed in the AKI or NAKI groups. Serum creatinine of patients in the AKI group decreased to baseline when discharged.

Urine samples were also collected from early DN (DN-micro, $\mathrm{n}=46)$ and overt DN (DNmacro, $n=34$ ) patients as the validation cohort between Feb 2019 and Aug 2019 in our hospital. The inclusion criteria were as follows: (1) Age ranged from 30 to 75 years; (2) eGFR >30 $\mathrm{mL} / \mathrm{min} / 1.73 \mathrm{~m}^{2}$; (3) Duration of T2DM > 5 years. Exclusion criteria were as follows: (1) Other etiology of CKD; (2) AKI within three months; (3) Acute and chronic inflammation of the

152 urinary system or other systems; (4) Patients with significant coexisting illnesses likely to affect survival or combined with other systemic diseases; (5) Patients with insufficient clinical data. 
154 The variables recorded for each participant included gender, age, body mass index (BMI),

155

156

157

158

159

160

161

162

163

164

165

166

167

168

169

170

171

172

173

174

175

176

177

178

179

180

181

182

183

184

185

186

187

188

189

190

191

192

193

systolic blood pressure (SBP), diastolic blood pressure (DBP), diabetes duration, medical history, hemoglobin, albumin, fasting blood glucose (FBG), hemoglobin Alc (HbA1c), lowdensity lipoprotein (LDL), high-density lipoprotein (HDL), total cholesterol (CHOL), triglyceride (TG), serum creatinine (Scr), uric acid (UA), blood urea nitrogen (BUN), urinary NGAL, and UAER.

All urine samples were collected in sterile tubes (Corning Incorporated, Corning, NY, USA) and placed immediately on ice. Cell debris of samples was removed by centrifugation (2000 rpm at $4^{\circ} \mathrm{C}$ for $12 \mathrm{~min}$ ). The supernatant was frozen at $-80^{\circ} \mathrm{C}$ for storage and subsequent extraction and analysis. All methods used in this study were approved by the Ethics Committee of the Second Xiangya Hospital, Central South University (IRB2017-S551). Written informed consent was collected from each patient before the study.

\section{Chemicals}

Methyl alcohol, acetonitrile, and ethyl alcohol were purchased from Merck Company, Germany. Milli-Q system (Millipore Corp., Bedford, MA, USA) ultrapure water was used throughout the study. Authentic standards were purchased from Sigma-Aldrich (St. Louis, MO, USA). Two standards of metabolites (trans-4-hydroxy-L-proline and azelaic acid) were purchased from Sigma-Aldrich. All chemicals were of analytical grade.

\section{Urine preparation for metabolite extraction}

The freeze-dried samples were crushed using a mixer mill (MM 400, Retsch) with a zirconia bead for $1.5 \mathrm{~min}$ at $30 \mathrm{~Hz}$. Then, $100 \mathrm{mg}$ of powder was extracted with $1.0 \mathrm{~mL} 70 \%$ aqueous methanol containing $0.1 \mathrm{mg} / \mathrm{L}$ lidocaine overnight at $4^{\circ} \mathrm{C}$. After $10000 \mathrm{~g}$ centrifugation for $10 \mathrm{~min}$, the supernatant was collected and filtered (SCAA-104, 0.22- $\mu \mathrm{m}$ pore size; ANPEL, Shanghai, China) prior to liquid chromatography-tandem mass spectrometry (LC-MS/MS) analysis. Quality Control (QC) samples were mixed by all samples to detect reproducibility of the whole experiment.

\section{Untargeted metabolomics analysis of the pilot study}

The extracted preoperative and postoperative urine samples from 12 patients were used in the pilot study. An LC-ESI-MS/MS system (UPLC, Shim-pack UFLC SHIMADZU CBM30A; MS/MS, Applied Biosystems 6500 QTRAP) were used to analyze the extracted compounds (Chen et al. 2013b). For analysis, 2- $\mu \mathrm{L}$ samples were injected onto a Waters ACQUITY UPLC HSS T3 C18 column $(2.1 \mathrm{~mm} * 100 \mathrm{~mm}, 1.8 \mu \mathrm{m})$ operated at $40^{\circ} \mathrm{C}$. The mobile phases used were $0.04 \%$ acidified water (Phase A) and $0.04 \%$ acidified acetonitrile (Phase B). Through the following gradient program process, compounds were effectively separated: $5 \% \mathrm{~B}$ at $0 \mathrm{~min} ; 95 \%$ $\mathrm{B}$ at $11.0 \mathrm{~min} ; 95 \% \mathrm{~B}$ at $12.0 \mathrm{~min} ; 5 \% \mathrm{~B}$ at $12.1 \mathrm{~min}$; and $5 \% \mathrm{~B}$ at $15.0 \mathrm{~min}$. The flow rate was $0.4 \mathrm{~mL} / \mathrm{min}$. The parameters used in the analysis are as follows: $500^{\circ} \mathrm{C}$ electrospray ionization (ESI) source temperature; $5500 \mathrm{~V}$ ion spray voltage (IS); 25 psi curtain gas (CUR); the collisionactivated dissociation (CAD) was set the highest. QQQ scans were acquired as multiple reaction monitoring (MRM) experiments with optimized declustering potential (DP) and collision energy (CE) for each individual MRM transition. The m/z range was set between 50 and 1000. 
194

195

196

197

198

199

200

201

202

203

204

205

206

207

208

209

210

211

212

213

214

215

216

217

218

219

220

221

222

223

224

225

226

227

228

229

230

231

232

233

Data filtering, peak detection, alignment, and calculations were performed using Analyst 1.6.1 software (AB Sciex). Signal/noise $>10$ peaks were checked manually. In-house software written in Perl was used to remove redundant signals. Accurate $\mathrm{m} / \mathrm{z}$ for each Q1 was acquired to identify the metabolites. Total ion chromatograms (TICs) and extracted ion chromatograms (EICs or XICs) of QC samples were used to construct metabolite profiles of all samples. MassBank, KNApSAcK, HMDB (Wishart et al. 2013), MoTo DB, and METLIN (Zhu et al. 2013) were the internal databases and public databases used to identify metabolites. The $\mathrm{m} / \mathrm{z}$ values, RT, and fragmentation patterns of metabolites were compared with standards.

\section{Targeted metabolomics analysis of the validation study}

Urine samples collected from 38 NAKI patients ( $24 \mathrm{~h}$ after operation), 38 AKI patients (24 h after operation), 46 early DN patients, and 34 overt DN patients were used in the validation study. Each urine sample was thawed on ice. Methanol was added to urine sample $(3: 1, \mathrm{v} / \mathrm{v})$, then vortex-mixed for $30 \mathrm{~s}$ and centrifuged at $12000 \mathrm{rpm}$ for $15 \mathrm{~min}$ at $4^{\circ} \mathrm{C}$. The supernatant was collected and placed at $-40^{\circ} \mathrm{C}$ for $1 \mathrm{~h}$. After centrifugation $\left(12000 \mathrm{rpm}\right.$ for $15 \mathrm{~min}$ at $4^{\circ} \mathrm{C}$ ), supernatant was placed in the vial for analysis. Standard stock solutions of trans-4-hydroxy-Lproline and azelaic acid were prepared in water/methanol $(1: 1, \mathrm{v} / \mathrm{v})$ at the concentration of 1 $\mathrm{mg} / \mathrm{mL}$. A series of trans-4-hydroxy-L-proline standards was prepared at the following concentration levels: 10, 20, 50, 100, 200 and $500 \mathrm{ng} / \mathrm{mL}$. A series of azelaic acid standards was prepared at the following concentration levels: 20, 50,100, 200, 500, 1000, and $2000 \mathrm{ng} / \mathrm{mL}$. Gradient diluted standards were used to establish standard curves.

Quantitative analysis was performed using an LC-ESI-MS/MS system (Waters Acquity UPLC; MS/MS: Applied Biosystems API 5500 QQQ-MS). 4- $\mu$ L prepared samples were applied to the ACQUITY UPLC BEH T3 column $(100 * 2.1 \mathrm{~mm}, 1.7 \mu \mathrm{m})$ at $40^{\circ} \mathrm{C}$. The mobile phases were composed of solvent A (water with $0.02 \%$ formic acid) and solvent B (acetonitrile), and the following gradient elution conditions were used with a flow rate of $0.35 \mathrm{~mL} / \mathrm{min}: 5 \% \mathrm{~B}$ at $0 \mathrm{~min}$; $10 \% \mathrm{~B}$ at $1.0 \mathrm{~min} ; 70 \% \mathrm{~B}$ at $2.0 \mathrm{~min} ; 90 \% \mathrm{~B}$ at $3 \mathrm{~min} ; 90 \% \mathrm{~B}$ at $4.0 \mathrm{~min} ; 10 \% \mathrm{~B}$ at $4.01 \mathrm{~min}$; $10 \% \mathrm{~B}$ at $5.0 \mathrm{~min}$. The following parameters were set for the mass spectrometer: temperature $550^{\circ} \mathrm{C}$, ion spray voltage $5500 \mathrm{~V}$, CUR $35 \mathrm{arb}, \mathrm{CAD} 7 \mathrm{arb}$, ionsource GS1 $55 \mathrm{arb}$, and ionsource GS2 55 arb. According to the above conditions, the standard solutions were sampled separately. Retention time of trans-4-hydroxy-L-proline was $0.68 \mathrm{~min}$, and azelaic acid was $2.53 \mathrm{~min}$. Standard curves were generated by plotting peak areas of the mass chromatogram (y) versus the concentration ( $\mathrm{x}$ ) and fitting to a linear regression (Trans-4-hydroxy-L-proline, $\mathrm{y}=136.32 \mathrm{x}$ 686.72, $\mathrm{R} 2=0.9997$; azelaic acid, $\mathrm{y}=6888.3 \mathrm{x}+311038, \mathrm{R} 2=0.9967)$. The identification of target metabolites in urine sample was based on the spectral pattern and retention time. Trans-4hydroxy-L-proline and azelaic acid were quantified based on their peak areas and standard curves.

The methods were validated based on parameters specified for bioanalytical methods according to U.S. Food and Drug Administration document and other related guidelines (Gonzalez et al. 2014). Selectivity, limit of detection (LOD) and limit of quantification (LOQ), linearity, precision and accuracy were evaluated. The LOD was defined as the concentration at a 
234 signal-to-noise ratio ( $\mathrm{S} / \mathrm{N}$ ) of $\geq 3$, and the $\mathrm{LOQ}$ was defined at $\mathrm{S} / \mathrm{N} \geq 10$ with precision lower 235 than $20 \%$. Each sample was analyzed in biological replicates of three and technical replicates of 236 three in this experiment.

237

238

239

240

241

242

243

244

245

246

247

248

249

250

251

252

253

254

255

256

257

258

259

260

261

262

263

264

265

266

267

268

269

270

271

272

273

\section{Data Analysis}

SPSS 23.0 software (SPSS Inc., Chicago, IL, USA) and GraphPad Prism 6.0 software were used for statistical analysis. Continuous variables were expressed as the means $\pm \mathrm{SD}$ and were compared using a Student's $t$ test, a Wilcoxon test, one-way ANOVA or receiver operating characteristic (ROC) curves analysis. Categorical data were expressed as percentages (\%) where appropriate and analyzed using Chi-square tests or Fisher's exact test. Statistical significance was considered if two-sided $\mathrm{p}<0.05$. Multivariate principal component analysis (PCA), partial least squares discriminant analysis (PLS-DA), orthogonal projection to latent structuresdiscriminant analysis (OPLS-DA), loadings plot and T-test, and univariate ANOVA were carried out to determine the differential metabolites between groups. A variable importance in projection (VIP) value of OPLS-DA model was applied to rank the metabolites that best distinguished between groups. Metabolites between two groups were considered different when the p-value of the T-test $<0.05$ and the variable importance in projection (VIP) $\geq 1$. Metabolites were mapped to the Kyoto Encyclopedia of Genes and Genomes (KEGG) database to determine important metabolic pathways and enrichment analysis. The calculated $\mathrm{p}$-value was validated through false discovery rate (FDR) correction, taking FDR $\leq 0.05$ as a threshold.

\section{Results}

\section{Participant characteristics}

This study was carried out in two stages, as shown in Figure 1. Clinical characteristics of all study patients are summarized in Table 1. A group of 88 T2DM inpatients, who underwent the elective off-pump CABG procedure, were enrolled in our study. The mean age of the study population was $59.15 \pm 8.76$ years, and $50 \%$ were male. The mean eGFR was $92.28 \pm 17.67$ $\mathrm{mL} / \mathrm{min} \cdot 1.73 \mathrm{~m}^{2}$. The mean diabetes duration was $10.00 \pm 5.12$ years. Urine samples were collected from these patients before and $24 \mathrm{~h}$ after CABG. Among the 44 patients in the AKI group, $70.5 \%$ were in AKI stage 1, 22.7\% were in AKI stage 2, and only $6.8 \%$ were in AKI stage 3. Six patients from the AKI group and six patients from the NAKI group were chosen as the pilot cohort. The preoperative clinical characteristics of two groups were matched. After operation, as expected, Scr and UNGAL in the AKI group were significantly higher than that in the NAKI group (Scr, $\mathrm{p}=0.001$; uNGAL, $\mathrm{p}=0.002$ ). The untargeted metabolomics analysis on the pilot cohort was aimed to identify biomarkers of postoperative AKI among T2DM patients. Two significantly altered metabolites found in untargeted metabolomics were quantified in the validation cohort by targeted metabolomics analysis. Postoperative urine samples from 38 NAKI patients and 38 AKI patients, urine samples from 46 early DN patients and 34 overt DN patients were used in the validation phase. Among the validation cohort, around $20 \%$ of T2DM patients receiving the CABG procedure had diabetic retinopathy, while more than $60 \%$ of DN patients had diabetic retinopathy. In addition, $26 \%$ early DN patients and $29.4 \%$ overt DN patients had a

Peer] reviewing PDF | (2020:01:45338:2:0:NEW 6 Apr 2020) 
274 history of ischemic heart disease. Age, gender, diabetes duration, BMI, FBG, and HbAlc were

275

276

277

278

279

280

281

282

283

284

285

286

287

288

289

290

291

292

293

294

295

296

297

298

299

300

301

302

303

304

305

306

307

308

309

310

311

312

313

not significantly different between the four groups. SBP, TG, UAER and number of patients with anemia were significantly higher in the DN-macro group, compared with the other three $(\mathrm{p}<$ 0.001). Renal function (BUN, Scr, eGFR) of the AKI group was the worst, followed by DNmacro, DN-micro, and NAKI groups $(\mathrm{p}<0.001)$.

\section{Results of untargeted metabolomics in the pilot cohort}

The stability of mass signals and accuracy

Metabolomics showed very stable performance as TIC chromatograms from all QC samples were overlapping in both positive ion and negative ion models (Supplementary Figure 1). Each of the different colored peaks in positive ion (Supplementary Figure 2A) and negative ion models (Supplementary Figure 2B) represents one MRM metabolite detected in the urine specimens. As it shown in Figure 2, the QC samples were tightly clustered in PCA scores plot, indicating high stability and reproducibility in the whole run. LC-MS/MS analysis of the discovery cohort urine samples identified a total of 742 known metabolites.

Metabolic profiling of preoperative samples and postoperative samples

The metabolic profiling of the A group and B group was evaluated to explore the postoperative differential metabolites among DM. The PCA score plot indicated the separation between the A group and B group in the discovery set (Figure 3A). The OPLS-DA model was constructed to obtain better discrimination between the above groups. As shown in Figure 3B, each code represents a tested urine sample. The loading plot of OPLS-DA clearly separated tested samples into two blocks according to their metabolic profiles of different groups. These results suggest that the biochemical metabolites in urine changed significantly after the CABG procedure among DM patients. According to the p-value of the T-test, combined with VIP values of the OPLS-DA model, 61 metabolites were statistically distinguishable between the two groups ( $\mathrm{R} 2 \mathrm{X}=0.407, \mathrm{R} 2 \mathrm{Y}=0.908, \mathrm{Q} 2=0.836$ ) and are shown in Supplementary Table. In Figure 3C, heat map analysis shows significantly different metabolites in the urine samples of the A and B groups, with 25 being presented at higher levels and 36 at lower levels in the B group compared with A group. In total, 22 pathways of KEGG classification were identified from 61 differential metabolites. KEGG pathway annotation showed that differentially expressed metabolites in DM patients after CABG were enriched in carbohydrate and amino acid metabolisms (Figure 3D). The functional mechanism of selected metabolomics was based on the pathway enrichment analysis using KEGG. The top 20 pathways are shown in Figure 3E. The top five metabolic pathways with the highest $\mathrm{p}$-values in the metabolic pathway enrichment analysis were phenylalanine metabolism, galactose metabolism, citrate cycle, prolactin signaling pathway, and proximal tubule bicarbonate reclamation.

\section{Screening differential metabolites related to postoperative AKI in DM}

We analyzed another two sets of pairwise data to identify AKI-related metabolites among DM patients: (i) AKI-B vs. AKI-A, (ii) AKI-B vs. NAKI-B. Two OPLS-DA models were constructed for these two comparisons. Figure 4A shows a clear separation between AKI-B and AKI-A. In total, 41 metabolites were differentially expressed in the AKI-B, compared with the

Peer) reviewing PDF | (2020:01:45338:2:0:NEW 6 Apr 2020) 
314 AKI-A group; of these, 18 were up-regulated and 23 were down-regulated (Figure 4B). Figure

$3154 \mathrm{C}$ shows a clear separation between AKI-B and NAKI-B. Moreover, 35 metabolites were

316 differentially expressed in the AKI-B group, compared with the NAKI-B group. Of these, 23

317 were up-regulated and 12 were down-regulated (Figure 4D).

318 The differential metabolites between AKI-B and AKI-A groups represent the

319 postoperative AKI-related metabolites. Because there were 41 metabolites in these data sets, it

320 was particularly important to select the metabolites with the greatest association with

321 postoperative AKI among DM patients. The data of B vs. A provided us with information about

322 operation-related differential metabolites. The data of AKI-B vs. NAKI-B provided us with

323 information about AKI-related differential metabolites. Through Venn analysis, the

324 postoperative AKI-related metabolites (the data of AKI-B vs. AKI-A) were compared with

325 operation-related metabolites (the data of B vs. A) and AKI-related metabolites (the data of AKI-

326 B vs. NAKI-B) to further reduce the number of postoperative AKI-related metabolites. Based on

327 the results of Venn analysis, the nine most significant markers of postoperative AKI were found.

328 The results show that the five up-regulated metabolites included trans-4-hydroxy-L-proline,

329 uridine triphosphate, $\mathrm{p}$-aminobenzoate, caffeic acid, and adrenochrome. The relative intensities

330 of these metabolites in the A and B groups are shown in Figure 5A-F. The four down-regulated

331 metabolites included $\delta$-valerolactam, L-norleucine, 5'-deoxy-5'-(methylthio) adenosine, and

332 azelaic acid. The relative intensities of these metabolites in the $\mathrm{A}$ and $\mathrm{B}$ groups are shown in

333 Figure 5G-K. These nine overlapping metabolites shown in Table 2 were mainly distributed in

334 the functional categories of amino acid, nucleotide, and organic acid metabolomics.

$335 \quad$ Validation of potential markers

336 The discovered metabolite markers trans-4-hydroxy-L-proline and azelaic acid, which

337 have the maximum absolute fold change value among the nine overlapping metabolites, were

338 further quantified in the validation cohort by targeted metabolomics analysis. To better

339 understand the possible role of these two metabolites in the acute and chronic development of

340 renal injury among T2DM patients, NAKI $(\mathrm{n}=38)$, AKI $(\mathrm{n}=38)$, DN-micro $(\mathrm{n}=46)$, and DN-

341 macro $(n=34)$ were chosen as the validation group. The results are shown in Figure 6 . The

342 absolute trans-4-hydroxy-L-proline concentration increased gradually from the NAKI group to

343 the AKI, DN-micro and DN-macro groups $(\mathrm{p}<0.0001)$ (Figure $6 \mathrm{~A})$. On the basis of quantitative

344 analysis results, ROC curves were applied to investigate the clinical diagnostic potentials of

345 these metabolites. An AUC value approaching 1.0 indicates a better diagnostic effectiveness. As

346 shown in Figure 6B-C, the AUC of trans-4-hydroxy-L-proline between AKI and DN-micro

347 groups was 0.722 (Figure 6B, $\mathrm{P}=0.0005,95 \% \mathrm{CI} 0.612-0.833$ ). The AUC of trans-4-hydroxy-L-

348 proline between $\mathrm{AKI}$ and $\mathrm{DN}$-macro groups was 0.783 (Figure $6 \mathrm{C}, \mathrm{P}<0.0001,95 \% \mathrm{CI} 0.669$ -

349 0.898). These data suggest that the abundances of urinary trans-4-hydroxy-L-proline increased

350 from acute renal damage to chronic renal damage in DM. For azelaic acid, the highest level was

351 found in the NAKI and DN-micro groups, followed by the DN-macro group. The lowest level

352 was found in the AKI group ( $p<0.0001$, Figure 6D). The azelaic acid concentrations in the

353 validation cohort were positively correlated with eGFR levels $(\mathrm{p}<0.0001)$, and the Pearson 
354 correlation coefficient was 0.539 (Figure 6E). As shown in Figure 6F-G, the AUC of azelaic acid

355

356

357

358

359

360

361

362

363

364

365

366

367

368

369

370

371

372

373

374

375

376

377

378

379

380

381

382

383

384

385

386

387

388

389

390

391

392

393

between NAKI and AKI groups was 0.860 (Figure 6F, $\mathrm{P}<0.0001,95 \% \mathrm{CI} 0.770-0.949$ ). The AUC of azelaic acid between DN-micro and DN-macro groups was 0.754 (Figure 6G, $\mathrm{P}=$ $0.0001,95 \% \mathrm{CI} 0.648-0.860$ ). These data suggest that the abundances of urinary azelaic acid decreased with acute or chronic deterioration of renal function in DM.

\section{Discussion}

Many studies reported that DM increases the risk of CABG postoperative complication (Brush et al. 2019; Hallberg et al. 2014; Shafranskaya et al. 2015). However, this is the first study to use untargeted metabolomics to exam metabolic changes of DM patients who experience operation-like acute attack. Via screening these operation-related metabolites, we identified some novel postoperative AKI markers of DM, and we further proved their role in the chronic progression of DN via targeted metabolomic analysis.

A prospective nested case-control study reported that after cardiopulmonary bypass surgery, all patients (with and without AKI) experienced tubular injury and stress, evidenced by proteinuria and the appearance of 2-microglobulinuria (Ho et al. 2009). In order to study the general metabolic changes of DM patients after CABG surgery, we enrolled T2DM inpatients who underwent the CABG procedure in our hospital. Six patients from the AKI group and six patients from the NAKI group were chosen as the pilot cohort. Via untargeted metabolomics analysis, we found the expression of 61 urine metabolites changed significantly after operation in all 12 patients (with and without AKI). Of interest, these operation-related metabolites in DM patients, which represent activated metabolic pathways in acute phase after operation, have been associated with chronic DN progression in previous reports.

We found urinary hippuric acid was elevated after operation, and indoxylsulfuric acid decreased after operation. Hippuric acid is converted from dietetic aromatic compounds by the gut microbiome, and high urinary hippuric acid had been linked to impaired glomerular filtration and tubular reabsorption (Liu et al. 2013). DN patients showed increased levels of urinary hippuric acid, compared with the healthy and T2DM patients (Li et al. 2017). Indoxylsulfuric acid is one of the uremic toxins derived from dietary proteins. In DM patients and animal models of DN, serum indoxylsulfuric acid levels increased with disease progression, and this was accompanied by the progression of renal function impairment (Atoh et al. 2009).

The tricarboxylic acid (TCA) cycle coordinates glucose, fatty acid, and amino acid metabolism. Key TCA cycle metabolites include lactate, pyruvate, citric acid, a-ketoglutaric acid, succinate, fumarate, and malate. We found after the CABG procedure, urinary citric acid significantly increased, whereas a-ketoglutaric acid significantly decreased. Elevated urinary TCA cycle metabolites have been observed in $\mathrm{db} / \mathrm{db}$ mice previously. Changes in urinary TCA cycle intermediates were also reported in $\mathrm{DN}$ participants who experienced progressive $\mathrm{CKD}$, compared with those with stable renal function (Li et al. 2013; Liu et al. 2018).

Urinary aromatic AA-tyrosine decreased after the CABG procedure in DM patients. Changes in urine tyrosine have been reported in T2DM patients with micro-macro albuminuria, 
394 compared with healthy and DM patients (Chen et al. 2018a; Pena et al. 2014). Besides the

395

396

397

398

399

400

401

402

403

404

405

406

407

408

409

410

411

412

413

414

415

416

417

418

419

420

421

422

423

424

425

426

427

428

429

430

431

432

433

metabolites mentioned above, trimethylamine $\mathrm{N}$-oxide, azelaic acid and sugars, including Dsorbitol and D-glucose, also changed after the operation in our study. These changes have been reported in association with chronic DN progression in other studies (Chen et al. 2013a; Hirayama et al. 2012; Klein \& Shearer 2016; Selby et al. 1995; Winther et al. 2019). In our study, some of our operation-related metabolites overlapped with some chronic DN progression metabolites reported in other studies. This indicated that, for DM patients, the pathological changes after acute events, such as an operation, can be detected by metabolomics and may be involved in the chronic progression of $\mathrm{DN}$.

DM is a known risk factor of AKI after cardiovascular surgery (Hertzberg et al. 2015). What effect does AKI occurring in DM will exert to kidney? The ischemic AKI induces significant interstitial inflammation and functional impairment/structural damage of small peritubular and glomerular blood vessels. Ischemic AKI in DM can further aggravate renal chronic inflammation and micovascular damage, which may affect the kidney in the long-term (He et al. 2017; Kelly et al. 2009; Patschan \& Muller 2016). Via non-invasive metabolomics of urine samples, we have realized the dynamic observation of short-term and long-term effects of AKI occurring in DM on kidney. After screening operation-related metabolites, we found 9 of 61 were postoperative AKI-related metabolites, including trans-4-hydroxy-L-proline, uridine triphosphate, p-aminobenzoate, caffeic acid, adrenochrome, $\delta$-valerolactam, L-norleucine, 5 'deoxy-5'-(methylthio) adenosine, and azelaic acid. Expression of these metabolites represents a single episode of kidney injury happen on DM. We speculate that each episode of kidney injury has a cumulative dose-response association. Several episodes may gradually accumulate and partially contribute to chronic DN progression. Among the nine metabolites, we choose two metabolites with the maximum absolute fold change value, trans-4-hydroxy-L-proline and azelaic acid, to verify our speculation by the validation cohort.

Trans-4-hydroxy-L-proline is one isomeric form of hydroxyproline, which is mainly identified in collagen, and it was found to be a good indicator of collagen metabolism in bone disease. Trans-4-hydroxy-L-proline is an important constituent of collagen and is a collagenspecific amino acid, with approximately $1 \%$ of the amino acid found in elastin (Srivastava et al. 2016). Its level in circulation has been correlated with the degree of fibrosis in chronic hepatitis (Attallah et al. 2007; Lawrence et al. 2019). Moreover, abnormal plasma trans-4-hydroxy-Lproline concentration was found in an insulin-dysregulated animal model (Kenez et al. 2018). Changes in trans-4-hydroxy-L-proline in circulation have been reported, along with collagen metabolism, fibrosis, and insulin-dysregulation. These changes seem to be related to DN progression, but no study has validated the contribution of trans-4-hydroxy-L-proline to early DN progression. In the validation cohort, we found urinary trans-4-hydroxy-L-proline concentration gradually increased with chronic DN progression, along with AKI episode. It is reported that every ischemic AKI insult diminishes the intrarenal total vascular surface area, subsequently followed or accompanied by endothelial-to-mesenchymal transdifferentiation (EndoMT) (O'Riordan et al. 2007; Zeisberg et al. 2008). EndoMT and epithelial-mesenchymal

Peer) reviewing PDF | (2020:01:45338:2:0:NEW 6 Apr 2020) 
434 transition (EMT) was associated with increased collagen production and renal fibrosis (Cruz-

435 Solbes \& Youker 2017; Zeng et al. 2019). As an indicator of collagen metabolism, we speculated

436 that the increase in urinary trans-4-hydroxy-L-proline after AKI may reflect mild renal fibrosis,

437 which may be undetectable by normal assay and could be treated as an early warning of DN

438 progression. With DN progression, AKI-like episodes and renal fibrosis increased, presenting as

439 an accumulation of urinary trans-4-hydroxy-L-proline concentration.

440

441

442

Azelaic acid is a saturated $\mathrm{C} 9$ dicarboxylic acid derived from the oxidation of fatty acids. Azelaic acid inhibits the generation of reactive oxygen species on neutrophils (Akamatsu et al. 1991). As a medicine, it possesses radical scavenging (Passi et al. 1991), antimicrobial

443 (Charnock et al. 2004), and antitumor (Breathnach 1999) potentials. Azelaic acid was found to

445 be effective in regulating high fat diet-induced oxidative stress and treating insulin resistance associated with T2DM in the mice model (Muthulakshmi et al. 2015). As a metabolite, azelaic acid was used to distinguish patients with depression and anxiety disorders from healthy controls (Chen et al. 2018b). Compared with healthy individuals, patients with psoriasis had lower azelaic acid levels (Kang et al. 2017). The levels of azelaic acid in serum were lower in DN patients, compared with non-DN patients (Hirayama et al. 2012). Our study found that the level of urinary azelaic acid cannot associate the acute renal events of DM with the chronic progression of DN, but it has a negative correlation with both acute and chronic deterioration of renal function in DM. Thus, urinary azelaic acid could be considered used to monitor renal function noninvasively among DM and DN patients.

We must consider the limitations of our study. First, the study was conducted in a singlecenter. The findings should be validated in a larger multicenter cohort. Second, only two of the nine metabolites were confirmed in the validation cohort. The other seven metabolites need future study. Third, association but not causal links can be described between trans-4-hydroxy-Lproline, azelaic acid, and DN progression. The precise molecular mechanisms underlying the results are still unknown. Mechanistic studies are needed to clarify the exact role of these metabolites in DN progression. Lastly, our results are limited to micro- and macro-albuminuria $\mathrm{DN}$, and it is necessary to test if our results apply in other conditions, such as the progression of DN to ESRD.

463

\section{Conclusion}

Our study explored the effect of AKI in DM on chronic DN progression and visualized this process via metabolomics. For DM patients, the pathological changes after acute events, such as operation, can be detected by metabolomics. The effect of the postoperative AKI on DM could be measured via, but not limited to, trans-4-hydroxy-L-proline and azelaic acid. The influence of AKI might accumulate and partially contribute to the chronic progression of DN. The detection of urinary trans-4-hydroxy-L-proline after AKI could be treated as an early warning of DN progression and might be linked to renal fibrosis. Meanwhile, urinary azelaic acid was identified as a noninvasive renal function indicator among DM and DN patients. Our results not only identified markers of AKI on DM and the chronic progression of DN but also 
474 interpreted the progression of $\mathrm{DN}$ from a new perspective. We associated chronic DN

475 progression with AKI occurring in DM, which might enable early monitoring and initiation of

476 specific therapies in patients with DM or DN.

477

478 Acknowledgements

479 Statement of Ethics

480 Studies have been approved by the appropriate institutional research ethics committee and have

481 been performed in accordance with the ethical standards as laid down in the 1964 Declaration of

482 Helsinki and its later amendments or comparable ethical standards.

\section{Disclosure Statement}

484 The authors have no conflicts of interest to declare.

485

486

487

488

489

490

491

492

493

494

495

496

497

498

499

500

501

502

503

504 
505

506

507

508

509

510

511

512

513

514

515

516

517

518

519

520

521

522

523

524

525

526

527

528

529

530

531

532

533

534

535

536

537

538

539

540

541

542

543

544

545

546

547

548

549

\section{References}

Akamatsu H, Komura J, Asada Y, Miyachi Y, and Niwa Y. 1991. Inhibitory effect of azelaic acid on neutrophil functions: a possible cause for its efficacy in treating pathogenetically unrelated diseases. Arch Dermatol Res 283:162-166. 10.1007/bf00372056

Arneth B, Arneth R, and Shams M. 2019. Metabolomics of Type 1 and Type 2 Diabetes. Int J Mol Sci 20. 10.3390/ijms20102467

Atoh K, Itoh H, and Haneda M. 2009. Serum indoxyl sulfate levels in patients with diabetic nephropathy: relation to renal function. Diabetes Res Clin Pract 83:220-226. 10.1016/j.diabres.2008.09.053

Attallah AM, Toson EA, Shiha GE, Omran MM, Abdel-Aziz MM, and El-Dosoky I. 2007. Evaluation of serum procollagen aminoterminal propeptide III, laminin, and hydroxyproline as predictors of severe fibrosis in patients with chronic hepatitis C. $J$ Immunoassay Immunochem 28:199-211. 10.1080/15321810701454649

Bellomo R, Auriemma S, Fabbri A, D'Onofrio A, Katz N, McCullough PA, Ricci Z, Shaw A, and Ronco C. 2008. The pathophysiology of cardiac surgery-associated acute kidney injury (CSA-AKI). Int J Artif Organs 31:166-178. 10.1177/039139880803100210

Breathnach AS. 1999. Azelaic acid: potential as a general antitumoural agent. Med Hypotheses 52:221-226. 10.1054/mehy.1997.0647

Brush JE, Jr., Siraj ES, Kemp CD, Liverman DP, McMichael BY, Lamichhane R, and Sheehan BE. 2019. Effect of Diabetes Mellitus on Complication Rates of Coronary Artery Bypass Grafting. Am J Cardiol 124:1389-1396. 10.1016/j.amjcard.2019.07.053

Charnock C, Brudeli B, and Klaveness J. 2004. Evaluation of the antibacterial efficacy of diesters of azelaic acid. Eur J Pharm Sci 21:589-596. 10.1016/j.ejps.2003.12.006

Chen C, Wang C, Hu C, Han Y, Zhao L, Zhu X, Xiao L, and Sun L. 2017. Normoalbuminuric diabetic kidney disease. Front Med 11:310-318. 10.1007/s11684-017-0542-7

Chen CJ, Liao WL, Chang CT, Lin YN, and Tsai FJ. 2018a. Identification of Urinary Metabolite Biomarkers of Type 2 Diabetes Nephropathy Using an Untargeted Metabolomic Approach. J Proteome Res 17:3997-4007. 10.1021/acs.jproteome.8b00644

Chen H, Li X, Yue R, Ren X, Zhang X, and Ni A. 2013a. The effects of diabetes mellitus and diabetic nephropathy on bone and mineral metabolism in T2DM patients. Diabetes Res Clin Pract 100:272-276. 10.1016/j.diabres.2013.03.007

Chen JJ, Bai SJ, Li WW, Zhou CJ, Zheng P, Fang L, Wang HY, Liu YY, and Xie P. 2018 b. Urinary biomarker panel for diagnosing patients with depression and anxiety disorders. Transl Psychiatry 8:192. 10.1038/s41398-018-0245-0

Chen W, Gong L, Guo Z, Wang W, Zhang H, Liu X, Yu S, Xiong L, and Luo J. 2013b. A novel integrated method for large-scale detection, identification, and quantification of widely targeted metabolites: application in the study of rice metabolomics. Mol Plant 6:17691780. $10.1093 / \mathrm{mp} / \mathrm{sst} 080$

Cho NH, Shaw JE, Karuranga S, Huang Y, da Rocha Fernandes JD, Ohlrogge AW, and Malanda B. 2018. IDF Diabetes Atlas: Global estimates of diabetes prevalence for 2017 and projections for 2045. Diabetes Res Clin Pract 138:271-281.

10.1016/j.diabres.2018.02.023

Colhoun HM, and Marcovecchio ML. 2018. Biomarkers of diabetic kidney disease. Diabetologia 61:996-1011. 10.1007/s00125-018-4567-5 
550

551

552

553

554

555

556

557

558

559

560

561

562

563

564

565

566

567

568

569

570

571

572

573

574

575

576

577

578

579

580

581

582

583

584

585

586

587

588

589

590

591

592

593

Cruz-Solbes AS, and Youker K. 2017. Epithelial to Mesenchymal Transition (EMT) and Endothelial to Mesenchymal Transition (EndMT): Role and Implications in Kidney Fibrosis. Results Probl Cell Differ 60:345-372. 10.1007/978-3-319-51436-9_13

Darshi M, Van Espen B, and Sharma K. 2016. Metabolomics in Diabetic Kidney Disease: Unraveling the Biochemistry of a Silent Killer. Am J Nephrol 44:92-103. $10.1159 / 000447954$

Gao G, Zhang B, Ramesh G, Betterly D, Tadagavadi RK, Wang W, and Reeves WB. 2013. TNF-alpha mediates increased susceptibility to ischemic AKI in diabetes. Am J Physiol Renal Physiol 304:F515-521. 10.1152/ajprenal.00533.2012

Girman CJ, Kou TD, Brodovicz K, Alexander CM, O'Neill EA, Engel S, Williams-Herman DE, and Katz L. 2012. Risk of acute renal failure in patients with Type 2 diabetes mellitus. Diabet Med 29:614-621. 10.1111/j.1464-5491.2011.03498.x

Gonzalez O, Blanco ME, Iriarte G, Bartolome L, Maguregui MI, and Alonso RM. 2014. Bioanalytical chromatographic method validation according to current regulations, with a special focus on the non-well defined parameters limit of quantification, robustness and matrix effect. J Chromatogr A 1353:10-27. 10.1016/j.chroma.2014.03.077

Hallan SI, Coresh J, Astor BC, Asberg A, Powe NR, Romundstad S, Hallan HA, Lydersen S, and Holmen J. 2006. International comparison of the relationship of chronic kidney disease prevalence and ESRD risk. J Am Soc Nephrol 17:2275-2284. 10.1681/ASN.2005121273

Hallberg V, Palomaki A, Lahtela J, Voutilainen S, Tarkka M, Kataja M, and Study G. 2014. Associations of metabolic syndrome and diabetes mellitus with 16-year survival after CABG. Cardiovasc Diabetol 13:25. 10.1186/1475-2840-13-25

He L, Wei Q, Liu J, Yi M, Liu Y, Liu H, Sun L, Peng Y, Liu F, Venkatachalam MA, and Dong Z. 2017. AKI on CKD: heightened injury, suppressed repair, and the underlying mechanisms. Kidney Int 92:1071-1083. 10.1016/j.kint.2017.06.030

Hertzberg D, Sartipy U, and Holzmann MJ. 2015. Type 1 and type 2 diabetes mellitus and risk of acute kidney injury after coronary artery bypass grafting. Am Heart $J$ 170:895-902. 10.1016/j.ahj.2015.08.013

Hirayama A, Nakashima E, Sugimoto M, Akiyama S, Sato W, Maruyama S, Matsuo S, Tomita M, Yuzawa Y, and Soga T. 2012. Metabolic profiling reveals new serum biomarkers for differentiating diabetic nephropathy. Anal Bioanal Chem 404:3101-3109. 10.1007/s00216-012-6412-x

Ho J, Lucy M, Krokhin O, Hayglass K, Pascoe E, Darroch G, Rush D, Nickerson P, Rigatto C, and Reslerova M. 2009. Mass spectrometry-based proteomic analysis of urine in acute kidney injury following cardiopulmonary bypass: a nested case-control study. Am J Kidney Dis 53:584-595. 10.1053/j.ajkd.2008.10.037

Kang H, Li X, Zhou Q, Quan C, Xue F, Zheng J, and Yu Y. 2017. Exploration of candidate biomarkers for human psoriasis based on gas chromatography-mass spectrometry serum metabolomics. Br J Dermatol 176:713-722. 10.1111/bjd.15008

Kellum JA, Lameire N, and Group KAGW. 2013. Diagnosis, evaluation, and management of acute kidney injury: a KDIGO summary (Part 1). Crit Care 17:204. 10.1186/cc11454

Kelly KJ, Burford JL, and Dominguez JH. 2009. Postischemic inflammatory syndrome: a critical mechanism of progression in diabetic nephropathy. Am J Physiol Renal Physiol 297:F923-931. 10.1152/ajprenal.00205.2009 
594

595

596

597

598

599

600

601

602

603

604

605

606

607

608

609

610

611

612

613

614

615

616

617

618

619

620

621

622

623

624

625

626

627

628

629

630

631

632

633

634

635

636

637

638
Kenez A, Warnken T, Feige K, and Huber K. 2018. Lower plasma trans-4-hydroxyproline and methionine sulfoxide levels are associated with insulin dysregulation in horses. BMC Vet Res 14:146. 10.1186/s12917-018-1479-z

Klein MS, and Shearer J. 2016. Metabolomics and Type 2 Diabetes: Translating Basic Research into Clinical Application. J Diabetes Res 2016:3898502. 10.1155/2016/3898502

Lawrence YA, Rodrigues-Hoffmann A, Steiner JM, Suchodolski JS, Shankar S, Klemashevich CL, and Lidbury JA. 2019. Development, validation, and application of a liquid chromatography-tandem mass spectrometry method for quantitative determination of trans-4-hydroxy-1-proline concentration in the serum of dogs with chronic hepatitis. Am J Vet Res 80:434-440. 10.2460/ajvr.80.5.434

Li L, Wang C, Yang H, Liu S, Lu Y, Fu P, and Liu J. 2017. Metabolomics reveal mitochondrial and fatty acid metabolism disorders that contribute to the development of DKD in T2DM patients. Mol Biosyst 13:2392-2400. 10.1039/c7mb00167c

Li M, Wang X, Aa J, Qin W, Zha W, Ge Y, Liu L, Zheng T, Cao B, Shi J, Zhao C, Wang X, Yu X, Wang G, and Liu Z. 2013. GC/TOFMS analysis of metabolites in serum and urine reveals metabolic perturbation of TCA cycle in $\mathrm{db} / \mathrm{db}$ mice involved in diabetic nephropathy. Am J Physiol Renal Physiol 304:F1317-1324. 10.1152/ajprenal.00536.2012

Liu J, Wang D, Chen Y, Sun H, He S, Wang C, Yang G, Shi M, Zhang J, Ren Y, Wang L, Lu Y, and Cheng J. 2013. 1H NMR-based metabonomic analysis of serum and urine in a nonhuman primate model of diabetic nephropathy. Mol Biosyst 9:2645-2652. $10.1039 / \mathrm{c} 3 \mathrm{mb} 70212 \mathrm{j}$

Liu JJ, Liu S, Gurung RL, Ching J, Kovalik JP, Tan TY, and Lim SC. 2018. Urine Tricarboxylic Acid Cycle Metabolites Predict Progressive Chronic Kidney Disease in Type 2 Diabetes. J Clin Endocrinol Metab 103:4357-4364. 10.1210/jc.2018-00947

Muthulakshmi S, Chakrabarti AK, and Mukherjee S. 2015. Gene expression profile of high-fat diet-fed C57BL/6J mice: in search of potential role of azelaic acid. J Physiol Biochem 71:29-42. 10.1007/s13105-014-0376-6

National Kidney F. 2002. K/DOQI clinical practice guidelines for chronic kidney disease: evaluation, classification, and stratification. Am J Kidney Dis 39:S1-266.

O'Riordan E, Mendelev N, Patschan S, Patschan D, Eskander J, Cohen-Gould L, Chander P, and Goligorsky MS. 2007. Chronic NOS inhibition actuates endothelial-mesenchymal transformation. Am J Physiol Heart Circ Physiol 292:H285-294. 10.1152/ajpheart.00560.2006

Passi S, Picardo M, De Luca C, Breathnach AS, and Nazzaro-Porro M. 1991. Scavenging activity of azelaic acid on hydroxyl radicals "in vitro". Free Radic Res Commun 11:329338. 10.3109/10715769109088931

Patschan D, and Muller GA. 2016. Acute Kidney Injury in Diabetes Mellitus. Int J Nephrol 2016:6232909. 10.1155/2016/6232909

Pena MJ, Lambers Heerspink HJ, Hellemons ME, Friedrich T, Dallmann G, Lajer M, Bakker SJ, Gansevoort RT, Rossing P, de Zeeuw D, and Roscioni SS. 2014. Urine and plasma metabolites predict the development of diabetic nephropathy in individuals with Type 2 diabetes mellitus. Diabet Med 31:1138-1147. 10.1111/dme.12447

Peng J, Li X, Zhang D, Chen JK, Su Y, Smith SB, and Dong Z. 2015. Hyperglycemia, p53, and mitochondrial pathway of apoptosis are involved in the susceptibility of diabetic models to ischemic acute kidney injury. Kidney Int 87:137-150. 10.1038/ki.2014.226

Peer) reviewing PDF | (2020:01:45338:2:0:NEW 6 Apr 2020) 
639

640

641

642

643

644

645

646

647

648

649

650

651

652

653

654

655

656

657

658

659

660

661

662

663

664

665

666

667

668

669

670

671

672

673

674

675

676

677

678

679

680

681

682

683

684

Rossi C, Marzano V, Consalvo A, Zucchelli M, Levi Mortera S, Casagrande V, Mavilio M, Sacchetta P, Federici M, Menghini R, Urbani A, and Ciavardelli D. 2018. Proteomic and metabolomic characterization of streptozotocin-induced diabetic nephropathy in TIMP3deficient mice. Acta Diabetol 55:121-129. 10.1007/s00592-017-1074-y

Selby PL, Shearing PA, and Marshall SM. 1995. Hydroxyproline excretion is increased in diabetes mellitus and related to the presence of microalbuminuria. Diabet Med 12:240243. 10.1111/j.1464-5491.1995.tb00465.x

Shafranskaya KS, Kashtalap VV, Kutikhin AG, Barbarash OL, and Barbarash LS. 2015. Microalbuminuria and prediction of cardiovascular complications in patients with coronary artery disease and type 2 diabetes mellitus after CABG surgery. Heart Lung Circ 24:951-959. 10.1016/j.hlc.2015.03.004

Srivastava AK, Khare P, Nagar HK, Raghuwanshi N, and Srivastava R. 2016. Hydroxyproline: A Potential Biochemical Marker and Its Role in the Pathogenesis of Different Diseases. Curr Protein Pept Sci 17:596-602.

Tabaei BP, Al-Kassab AS, Ilag LL, Zawacki CM, and Herman WH. 2001. Does microalbuminuria predict diabetic nephropathy? Diabetes Care 24:1560-1566. 10.2337/diacare.24.9.1560

Tang X, You J, Liu D, Xia M, He L, and Liu H. 2019. 5-Hydroxyhexanoic Acid Predicts Early Renal Functional Decline in Type 2 Diabetes Patients with Microalbuminuria. Kidney Blood Press Res 44:245-263. 10.1159/000498962

Wang TJ, Larson MG, Vasan RS, Cheng S, Rhee EP, McCabe E, Lewis GD, Fox CS, Jacques PF, Fernandez C, O'Donnell CJ, Carr SA, Mootha VK, Florez JC, Souza A, Melander O, Clish CB, and Gerszten RE. 2011. Metabolite profiles and the risk of developing diabetes. Nat Med 17:448-453. 10.1038/nm.2307

Winther SA, Ollgaard JC, Tofte N, Tarnow L, Wang Z, Ahluwalia TS, Jorsal A, Theilade S, Parving HH, Hansen TW, Hazen SL, Pedersen O, and Rossing P. 2019. Utility of Plasma Concentration of Trimethylamine N-Oxide in Predicting Cardiovascular and Renal Complications in Individuals With Type 1 Diabetes. Diabetes Care 42:1512-1520. $10.2337 / \mathrm{dc} 19-0048$

Wishart DS, Jewison T, Guo AC, Wilson M, Knox C, Liu Y, Djoumbou Y, Mandal R, Aziat F, Dong E, Bouatra S, Sinelnikov I, Arndt D, Xia J, Liu P, Yallou F, Bjorndahl T, PerezPineiro R, Eisner R, Allen F, Neveu V, Greiner R, and Scalbert A. 2013. HMDB 3.0-The Human Metabolome Database in 2013. Nucleic Acids Res 41:D801-807. 10.1093/nar/gks1065

Zeisberg EM, Potenta SE, Sugimoto H, Zeisberg M, and Kalluri R. 2008. Fibroblasts in kidney fibrosis emerge via endothelial-to-mesenchymal transition. J Am Soc Nephrol 19:22822287. 10.1681/ASN.2008050513

Zeng LF, Xiao Y, and Sun L. 2019. A Glimpse of the Mechanisms Related to Renal Fibrosis in Diabetic Nephropathy. Adv Exp Med Biol 1165:49-79. 10.1007/978-981-13-8871-2_4

Zhang J, Yan L, Chen W, Lin L, Song X, Yan X, Hang W, and Huang B. 2009. Metabonomics research of diabetic nephropathy and type 2 diabetes mellitus based on UPLC-oaTOFMS system. Anal Chim Acta 650:16-22. 10.1016/j.aca.2009.02.027

Zhu ZJ, Schultz AW, Wang J, Johnson CH, Yannone SM, Patti GJ, and Siuzdak G. 2013. Liquid chromatography quadrupole time-of-flight mass spectrometry characterization of metabolites guided by the METLIN database. Nat Protoc 8:451-460. 10.1038/nprot.2013.004

Peer] reviewing PDF | (2020:01:45338:2:0:NEW 6 Apr 2020) 


\section{Figure legends}

686

687

688

689

690

691

692

693

694

695

696

697

698

699

700

701

702

703

704

705

706

707

708

709

710

711

712

713

714

715

716

717

718

719

720

721

722

723

Figure 1. Flow diagram of study design

Abbreviations: CABG, coronary artery bypass grafting.

Figure 2. PCA performed on all samples

Urine samples in different groups are represented by differently colored symbols.

Abbreviations: PCA, principal components analysis.

Figure 3. Separating postoperative samples from preoperative samples by metabolic profiling analysis.

(A) Score scatter plot of PCA model for B group vs A group; (B) Score scatter plot of OPLS-DA model for B group vs A group; (C) Heat map representing statistically different metabolites among two groups. Scale and metabolites category are provided to right of heat map; (D) KEGG pathways annotation based on significant different metabolites between B vs A group. The $x$ axis indicates the proportion and number of metabolites annotated to the pathway, and the $y$ axis indicates name of the KEGG metabolic pathway; (E) Statistics of KEGG enrichment. The $x$ axis indicates the rich factor corresponding to each pathway, and the $y$ axis indicates name of the KEGG metabolic pathway. The size of bubble indicates number. The color of the point represents the $\mathrm{p}$-values of the enrichment analysis.

Abbreviations: PCA, principal components analysis; OPLS-DA, orthogonal projection to latent structures- discriminant analysis; A, preoperative sample; B, postoperative sample; KEGG, the Kyoto Encyclopedia of Genes and Genomes.

Figure 4. OPLS-DA score plot of of AKI-B vs. AKI-A and AKI-B vs. NAKI-B (A, B) OPLS-DA score plot of AKI-B vs AKI-A, eighteen and twenty-three up- and downregulated metabolites, respectively, in AKI-B group compared with the AKI-A group; (C, D) OPLS-DA score plot of AKI-B vs NAKI-B, twenty-three and twelve up- and down-regulated metabolites, respectively, in AKI-B group compared with the NAKI-B group.

Abbreviations: OPLS-DA, orthogonal projection to latent structures- discriminant analysis; A, preoperative sample; B, postoperative sample.

Figure 5. Venn analysis identified metabolites associated with postoperative AKI in DM (A) Venn diagram of the up-regulated overlapping metabolites from three pairs data (B vs. A, AKI-B vs. AKI-A and AKI-B vs. NAKI-B); (B-F) The relative intensities of the 5 up-regulated overlapping metabolites before and after $\mathrm{CABG} ;(\mathrm{G})$ Venn diagram of the down-regulated overlapping metabolites from three pairs data (B vs. A, AKI-B vs. AKI-A and AKI-B vs. NAKIB); (H-K) The relative intensities of the 4 down-regulated overlapping metabolites before and after $\mathrm{CABG}$.

Peer] reviewing PDF | (2020:01:45338:2:0:NEW 6 Apr 2020) 
724 Abbreviations: CABG, coronary artery bypass grafting; A, preoperative sample; B, postoperative

725

726

727

728

729

730

731

732

733

734

735

736

737

738

739

740

741

742

743

744

745

746

747

748 sample.

Figure 6. Urine metabolomic abundance in validation cohort were measured by targeted metabolomics analysis

(A) The absolute concentration levels of trans-4-hydroxy-L-proline in NAKI, AKI, DN-micro and DN-macro groups; (B-C) ROC curves of trans-4-hydroxy-L-proline for distinguishing AKI from DN-micro and DN-macro patients; The sensitivity (true positive rate) is set as the ordinate, and 1-specificity (false positive rate) is set as the abscissa. (D) The absolute concentration levels of azelaic acid in NAKI, AKI, DN-micro and DN-macro groups. (E) Correlation of azelaic acid with eGFR in cohort patients. Pearson correlation coefficients (R) is provided in; (F-G) ROC curves of azelaic acid for distinguishing NAKI from AKI patients, DN-micro from DN-macro patients. $\mathrm{p}<0.05$ was considered significant. ${ }^{*}, \mathrm{p}<0.05$ vs NAKI group; $\#, \mathrm{p}<0.05$ vs AKI group; \&, p $<0.05$ vs DN-micro group.

Abbreviation: ROC, receiver operating characteristic curves.

Supplementary Figure 1. TIC from the QC samples

Positive ionization mode (A) and negative ionization mode (B) of the typical TIC from the QC samples.

Abbreviation: TIC, total ion chromatograms; QC, Quality Control.

\section{Supplementary Figure 2. MRM spectra obtained from the QC samples}

MRM spectra obtained from the QC samples with positive (A) and negative mode (B).

Abbreviation: MRM, multiple reaction monitoring; QC, Quality Control. 


\section{Table $\mathbf{1}$ (on next page)}

Clinical characteristics of pilot and validation patients

Note: Data are presented as $\mathrm{N}(\%)$ or mean \pm SD. $\mathrm{p}<0.05$ was considered significant. $*, \mathrm{p}<$ 0.05 vs NAKI group; \#, $p<0.05$ vs AKI group; $\&, p<0.05$ vs DN-micro group. Abbreviations: BMI, body mass index; SBP, systolic blood pressure; DBP, diastolic blood pressure; FBG, fasting blood glucose; HbAlc, haemoglobin Alc; LDL, low-density lipoprotein; HDL, highdensity lipoprotein; $\mathrm{CHOL}$, total cholesterol; TG, triglyceride; eGFR, estimated glomerular filtration rate; Scr, serum creatinine; UA, uric acid; BUN, blood urea nitrogen; UAER, urinary albumin excretion rate. 
1 Table 1. Clinical characteristics of pilot and validation patients

2

3

\begin{tabular}{|c|c|c|c|c|c|c|c|c|}
\hline \multirow[b]{2}{*}{ Variables } & \multicolumn{3}{|c|}{ Pilot cohort } & \multicolumn{5}{|c|}{ Validation cohort } \\
\hline & NAKI $(n=6)$ & AKI $(n=6)$ & $p$ value & $\begin{array}{l}\text { NAKI } \\
(n=38)\end{array}$ & $\begin{array}{c}\text { AKI } \\
(n=38)\end{array}$ & $\begin{array}{l}\text { DN-micro } \\
(n=46)\end{array}$ & $\begin{array}{l}\text { DN-macro } \\
(n=34)\end{array}$ & $p$ value \\
\hline Age (years) & $59.00 \pm 6.13$ & $55.66 \pm 7.39$ & 0.415 & $60.28 \pm 9.39$ & $58.60 \pm 8.73$ & $58.13 \pm 10.29$ & $61.32 \pm 8.35$ & 0.406 \\
\hline Male (\%) & 50 & 50 & 1.000 & 47.4 & 52.6 & 52.2 & 58.8 & 0.822 \\
\hline DM duration (years) & $10.33 \pm 6.71$ & $9.66 \pm 6.28$ & 0.863 & $10.21 \pm 5.05$ & $9.78 \pm 4.96$ & $12.34 \pm 6.78$ & $11.85 \pm 5.53$ & 0.192 \\
\hline \multicolumn{9}{|l|}{ Medical history (\%) } \\
\hline Diabetic retinopathy & 16.7 & 16.7 & 1.000 & 18.4 & 21.1 & 69.6 & 67.6 & $<0.001$ \\
\hline Stroke & 0 & 0 & 1.000 & 5.3 & 7.9 & 8.7 & 11.8 & 0.799 \\
\hline BMI & $22.73 \pm 3.71$ & $25.60 \pm 4.28$ & 0.244 & $24.19 \pm 3.05$ & $25.13 \pm 2.74$ & $24.55 \pm 2.90$ & $24.74 \pm 2.46$ & 0.529 \\
\hline $\mathrm{SBP}(\mathrm{mm} \mathrm{Hg})$ & $138.50 \pm 30.98$ & $146 \pm 19.45$ & 0.619 & $145.44 \pm 21.82$ & $147.18 \pm 22.45$ & $145.28 \pm 20.29$ & $164.64 \pm 20.07^{*}, \#, \&$ & $<0.001$ \\
\hline $\mathrm{DBP}(\mathrm{mm} \mathrm{Hg})$ & $77.16 \pm 11.58$ & $83.33 \pm 6.94$ & 0.290 & $80.34 \pm 13.66$ & $81.55 \pm 8.68$ & $83.76 \pm 14.45$ & $87.73 \pm 15.54$ & 0.102 \\
\hline Serum albumin (g/L) & $42.06 \pm 2.79$ & $42.25 \pm 2.51$ & 0.907 & $42.30 \pm 4.20$ & $41.82 \pm 5.00$ & $37.01 \pm 4.85^{*}$, \# & $34.07 \pm 5.57 *, \#, \&$ & $<0.001$ \\
\hline Anemia (\%) & 16.7 & 16.7 & 1.000 & 13.2 & 10.5 & 19.6 & $35.3^{*}, \#, \&$ & 0.038 \\
\hline
\end{tabular}




\begin{tabular}{|c|c|c|c|c|c|c|c|c|}
\hline $\mathrm{FBG}(\mathrm{mmol} / \mathrm{L})$ & $6.84 \pm 1.23$ & $7.14 \pm 1.83$ & 0.748 & $7.07 \pm 2.97$ & $7.52 \pm 2.43$ & $7.66 \pm 2.15$ & $7.61 \pm 3.02$ & 0.752 \\
\hline HbA1c (\%) & $7.83 \pm 0.92$ & $7.58 \pm 1.01$ & 0.664 & $7.75 \pm 1.87$ & $8.36 \pm 2.22$ & $8.89 \pm 2.26$ & $8.76 \pm 2.10$ & 0.082 \\
\hline $\operatorname{Scr}(\mu \mathrm{mol} / \mathrm{L})$ & $76.65 \pm 12.98$ & $82.38 \pm 18.08$ & 0.542 & & & & & \\
\hline $\begin{array}{l}\text { Postoperative Scr } \\
\text { ( } \mu \mathrm{mol} / \mathrm{L})\end{array}$ & $79.91 \pm 10.61$ & $145.11 \pm 31.14$ & 0.001 & $76.02 \pm 17.08 \#$ & $139.93 \pm 46.16$ & $78.71 \pm 24.66 \#$ & $92.99 \pm 22.30 \#, \&$ & $<0.001$ \\
\hline BUN (mmol/L) & $6.18 \pm 1.06$ & $7.46 \pm 1.69$ & 0.150 & & & & & \\
\hline $\begin{array}{l}\text { Postoperative BUN } \\
\qquad(\mathrm{mmol} / \mathrm{L})\end{array}$ & $7.13 \pm 2.55$ & $10.01 \pm 3.27$ & 0.120 & $6.70 \pm 1.59 \#$ & $10.05 \pm 2.74$ & $6.79 \pm 2.16 \#$ & $7.64 \pm 2.13 \#$ & $<0.001$ \\
\hline $\mathrm{UA}(\mu \mathrm{mol} / \mathrm{L})$ & $419.23 \pm 138.82$ & $374.45 \pm 93.78$ & 0.527 & & & & & \\
\hline $\begin{array}{l}\text { Postoperative UA } \\
\text { ( } \mu \mathrm{mol} / \mathrm{L})\end{array}$ & $324.58 \pm 173.92$ & $375.00 \pm 84.70$ & 0.538 & $352.56 \pm 87.74$ & $358.85 \pm 82.61$ & $343.08 \pm 90.85$ & $304.36 \pm 72.08 \#$ & 0.034 \\
\hline $\begin{array}{c}\text { eGFR } \\
\left(\mathrm{mL} / \mathrm{min} \cdot 1.73 \mathrm{~m}^{2}\right) \\
\text { Postoperative eGFR } \\
\left(\mathrm{mL} / \mathrm{min} \cdot 1.73 \mathrm{~m}^{2}\right)\end{array}$ & $88.01 \pm 24.39$ & $85.80 \pm 25.30$ & 0.011 & $83.09 \pm 16.60 \#$ & $46.68 \pm 16.54$ & $86.14 \pm 25.00 \#$ & $72.91 \pm 19.36 \#, \&$ & $<0.001$ \\
\hline $\begin{array}{l}\text { Postoperative urinary } \\
\qquad \mathrm{NGAL}(\mu \mathrm{mol} / \mathrm{L})\end{array}$ & $15.75 \pm 14.55$ & $\begin{array}{l}324.39 \pm \\
134.46\end{array}$ & 0.002 & $12.84 \pm 15.44$ & $303.74 \pm 120.85$ & & & $<0.001$ \\
\hline
\end{tabular}




\begin{tabular}{ccccccccc}
\hline TG (mmol/L) & $1.71 \pm 0.53$ & $1.37 \pm 0.57$ & 0.302 & $1.74 \pm 0.65$ & $2.27 \pm 1.00$ & $2.10 \pm 0.93$ & $3.16 \pm 2.40^{*}, \#, \&$ & $<0.001$ \\
\hline CHOL (mmol/L) & $4.18 \pm 1.19$ & $3.95 \pm 0.64$ & 0.686 & $4.29 \pm 1.28$ & $5.03 \pm 1.30$ & $4.16 \pm 1.20 \#$ & $5.15 \pm 1.43^{*}, \&$ & 0.001 \\
\hline HDL (mmol/L) & $0.93 \pm 0.09$ & $1.10 \pm 0.16$ & 0.063 & $0.99 \pm 0.25$ & $1.05 \pm 0.32$ & $0.99 \pm 0.21$ & $1.09 \pm 0.25$ & 0.238 \\
\hline LDL (mmol/L) & $2.85 \pm 1.04$ & $2.40 \pm 0.60$ & 0.383 & $2.79 \pm 1.10$ & $3.24 \pm 1.01$ & $2.62 \pm 1.15$ & $3.35 \pm 1.31 \&$ & 0.012 \\
\hline UAER (mg/day) & $7.58 \pm 8.01$ & $8.13 \pm 9.96$ & 0.918 & $13.57 \pm 9.11$ & $12.41 \pm 9.07$ & $88.45 \pm 51.27$ & $1533.63 \pm 1096.98^{*}, \#, \& \quad<0.001$
\end{tabular}

4 Note: Data are presented as N (\%) or mean \pm SD. $p<0.05$ was considered significant. *, $p<0.05$ vs NAKI group; \#, $p<0.05$ vs AKI

5 group; $\&, p<0.05$ vs DN-micro group.

6 Abbreviations: BMI, body mass index; SBP, systolic blood pressure; DBP, diastolic blood pressure; FBG, fasting blood glucose; HbA1c,

7 haemoglobin A1c; LDL, low-density lipoprotein; HDL, high-density lipoprotein; CHOL, total cholesterol; TG, triglyceride; eGFR,

8 estimated glomerular filtration rate; Scr, serum creatinine; UA, uric acid; BUN, blood urea nitrogen; UAER, urinary albumin excretion

9 rate.

10 


\section{Table 2 (on next page)}

The nine urine overlapping metabolites potentially associated with postoperative AKI in DM.

Note: Metabolites between two groups were considered different when the p-value of the Ttest $<0.05$ and VIP $\geq 1$. Abbreviations: A, preoperative sample; B, postoperative sample; VIP, variable importance in projection. 
3 Table 2. The nine urine overlapping metabolites potentially associated with postoperative AKI in DM.

4

\begin{tabular}{|c|c|c|c|c|c|c|c|c|c|c|}
\hline \multirow{2}{*}{ Metabolite } & \multirow{2}{*}{$\begin{array}{l}\text { Change } \\
\text { trend }\end{array}$} & \multicolumn{3}{|c|}{ B vs. A } & \multicolumn{3}{|c|}{ AKI-B vs. AKI-A } & \multicolumn{3}{|c|}{ AKI-B vs. NAKI-B } \\
\hline & & $\begin{array}{l}\text { log2 fold } \\
\text { change }\end{array}$ & $p$ value & VIP & $\begin{array}{l}\text { log2 fold } \\
\text { change }\end{array}$ & $p$ value & VIP & $\begin{array}{l}\text { log2 fold } \\
\text { change }\end{array}$ & $p$ value & VIP \\
\hline Trans-4-hydroxy-L-proline & up & 6.426 & 0.001 & 1.991 & 6.912 & 0.005 & 2.376 & 3.513 & 0.003 & 5.468 \\
\hline Uridine triphosphate & up & 5.117 & 0.019 & 1.828 & 6.625 & 0.029 & 2.612 & 1.579 & 0.017 & $2.62 \varepsilon$ \\
\hline P-Aminobenzoate & up & 2.159 & 0.041 & 1.982 & 3.487 & 0.010 & 3.618 & 2.704 & 0.030 & 3.415 \\
\hline Caffeic Acid & up & 0.966 & 0.010 & 1.049 & 1.366 & 0.013 & 1.608 & 0.923 & 0.014 & 1.613 \\
\hline Adrenochrome & up & 0.791 & $<0.001$ & 2.624 & 1.203 & 0.013 & 4.182 & 0.765 & 0.024 & 3.760 \\
\hline$\delta$-Valerolactam & down & -0.744 & $<0.001$ & 2.819 & -1.149 & 0.004 & 2.429 & -1.455 & 0.032 & 5.574 \\
\hline L-Norleucine & down & -1.330 & 0.001 & 3.745 & -1.886 & 0.018 & 4.461 & -1.078 & 0.006 & 3.888 \\
\hline $\begin{array}{c}\text { 5'-Deoxy-5'-(Methylthio) } \\
\text { Adenosine }\end{array}$ & down & -1.519 & 0.016 & 1.057 & -1.938 & 0.020 & 1.065 & -1.244 & 0.008 & 1.223 \\
\hline Azelaic acid & down & -3.338 & $<0.001$ & 2.768 & -4.254 & 0.007 & 3.110 & -1.674 & 0.048 & 1.161 \\
\hline
\end{tabular}

7 Note: Metabolites between two groups were considered different when the $p$-value of the T-test $<0.05$ and VIP $\geq 1$.

8 Abbreviations: A, preoperative sample; B, postoperative sample; VIP, variable importance in projection. 
Figure 1

\section{Flow diagram of study design}

Abbreviations: CABG, coronary artery bypass grafting.

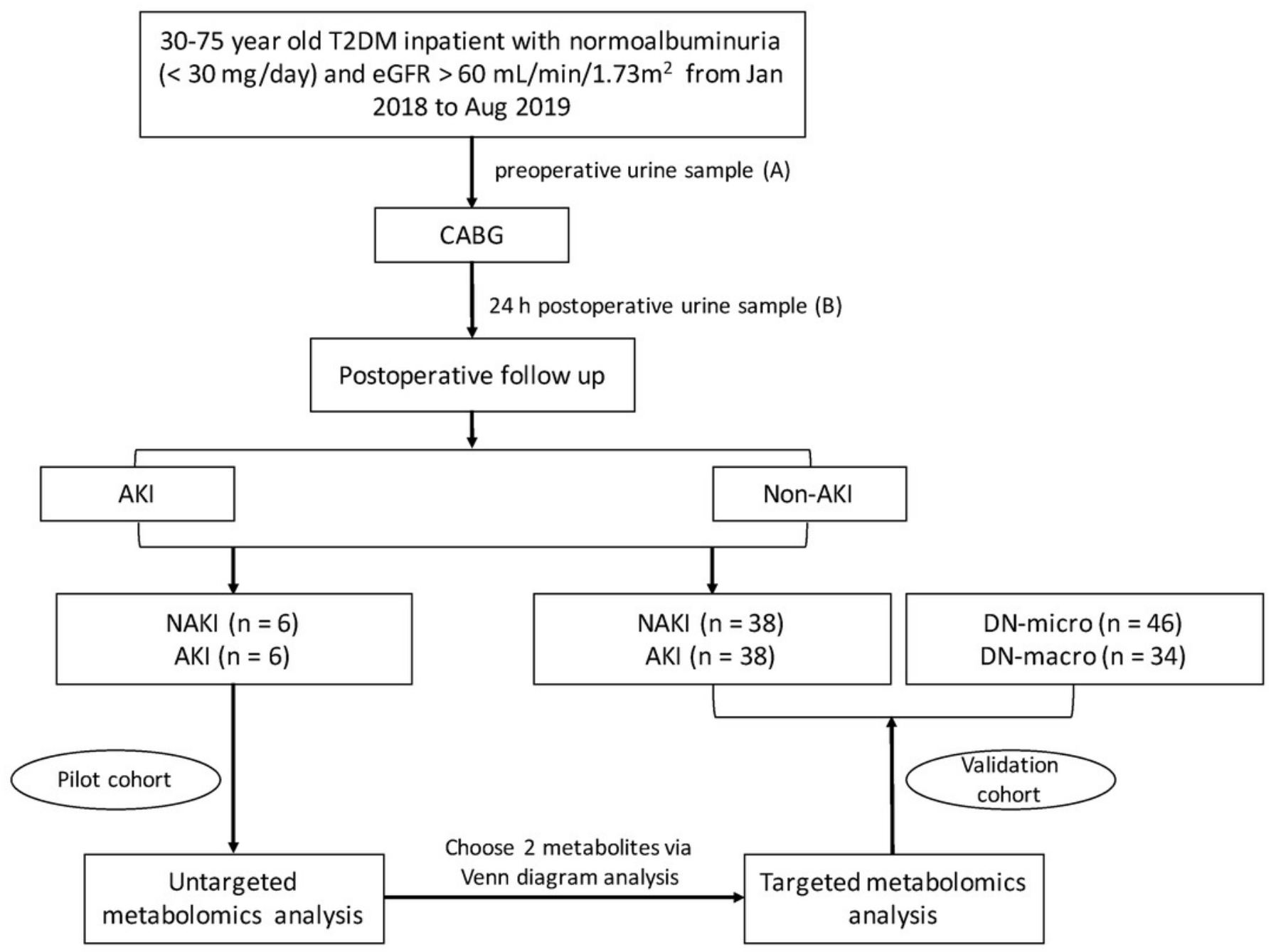


Figure 2

PCA performed on all samples

Urine samples in different groups are represented by differently colored symbols.

Abbreviations: PCA, principal components analysis.

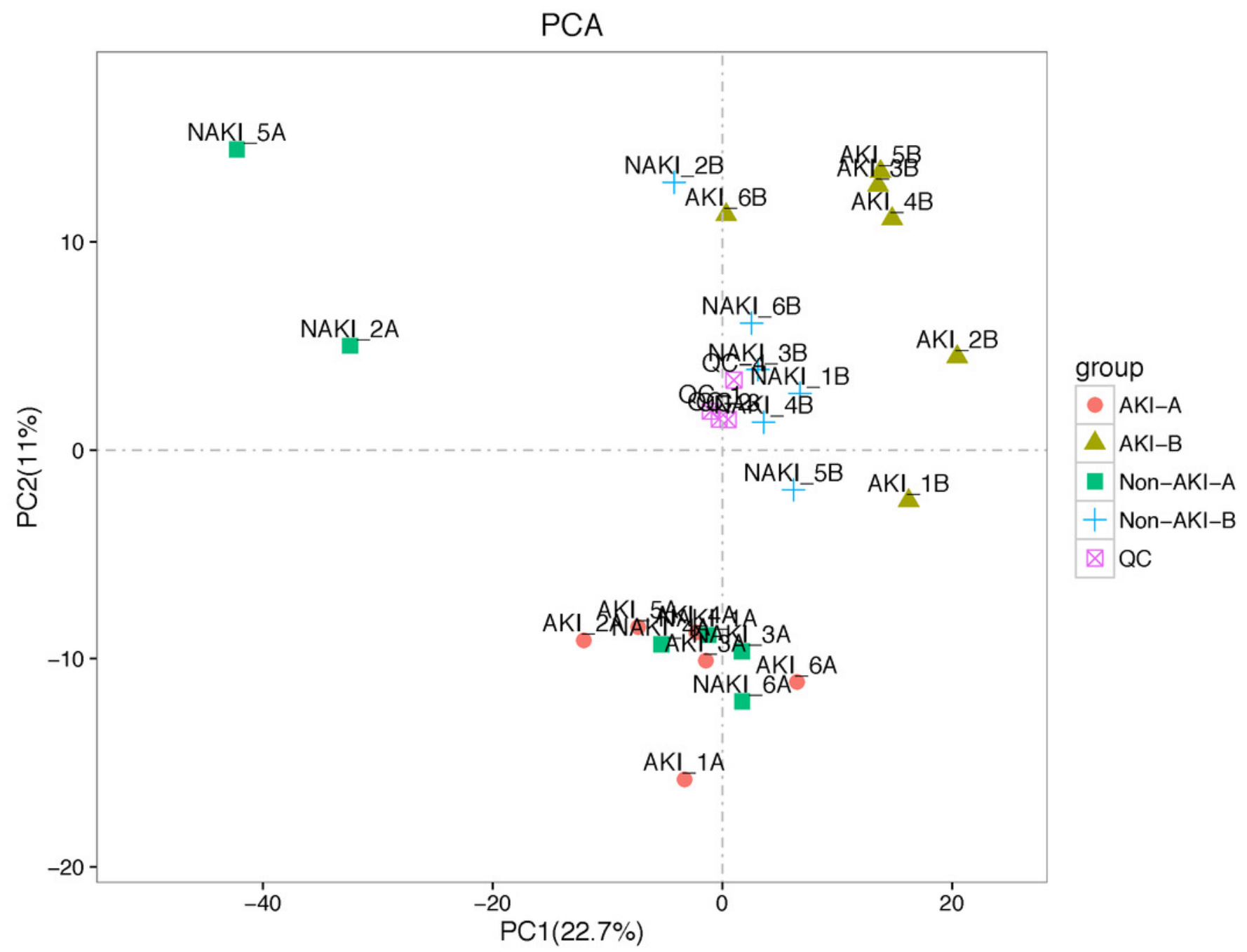




\section{Figure 3}

Separating postoperative samples from preoperative samples by metabolic profiling analysis.

(A) Score scatter plot of PCA model for B group vs A group; (B) Score scatter plot of OPLS-DA model for B group vs A group; (C) Heat map representing statistically different metabolites among two groups. Scale and metabolites category are provided to right of heat map; (D) KEGG pathways annotation based on significant different metabolites between B vs A group. The $x$ axis indicates the proportion and number of metabolites annotated to the pathway, and the $y$ axis indicates name of the KEGG metabolic pathway; (E) Statistics of KEGG enrichment. The $x$ axis indicates the rich factor corresponding to each pathway, and the $y$ axis indicates name of the KEGG metabolic pathway. The size of bubble indicates number. The color of the point represents the $p$-values of the enrichment analysis. Abbreviations: PCA, principal components analysis; OPLS-DA, orthogonal projection to latent structuresdiscriminant analysis; A, preoperative sample; B, postoperative sample; KEGG, the Kyoto Encyclopedia of Genes and Genomes. 
A

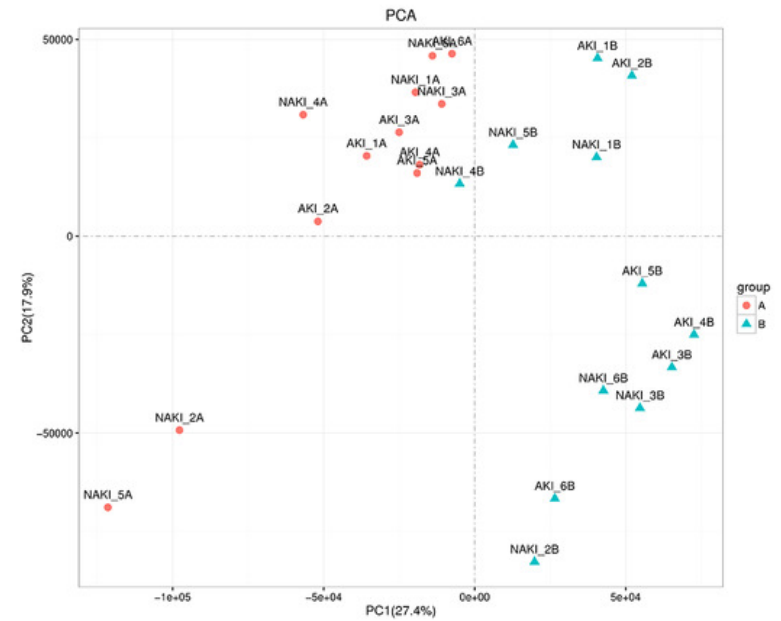

B

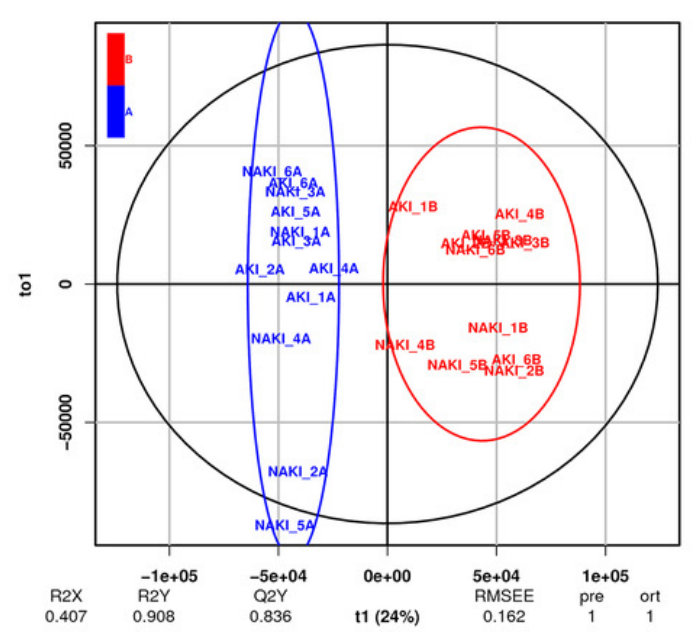

C

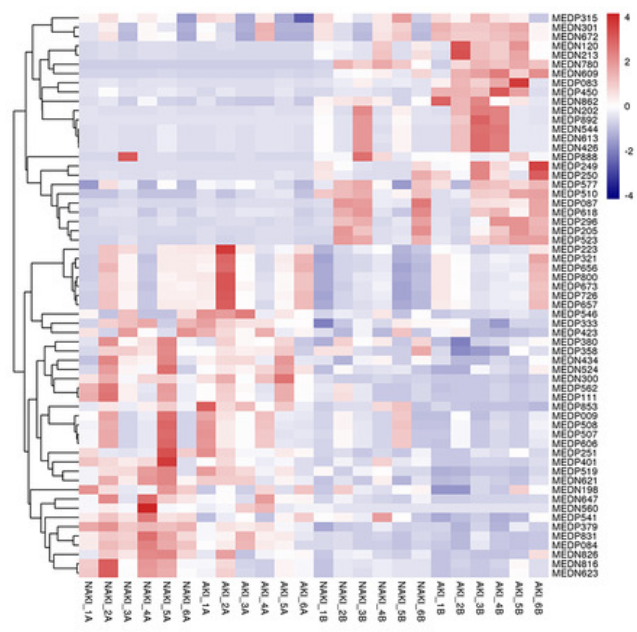

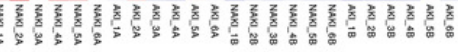

D

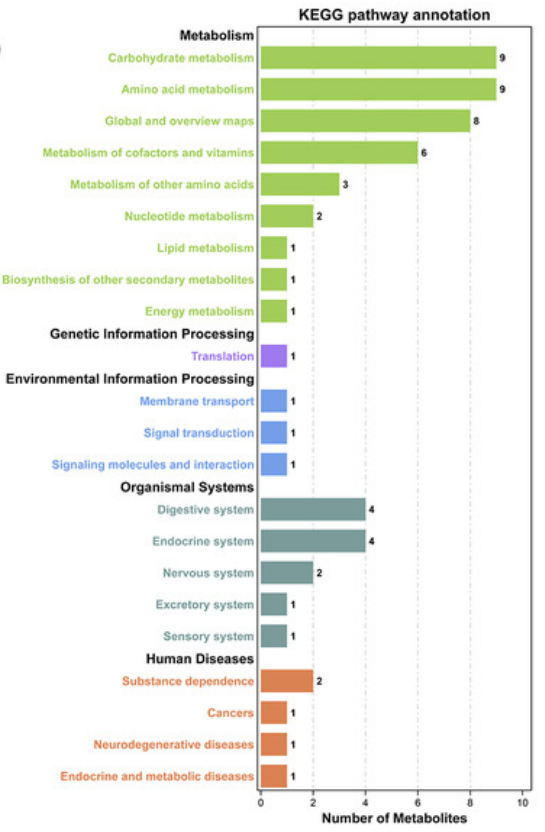

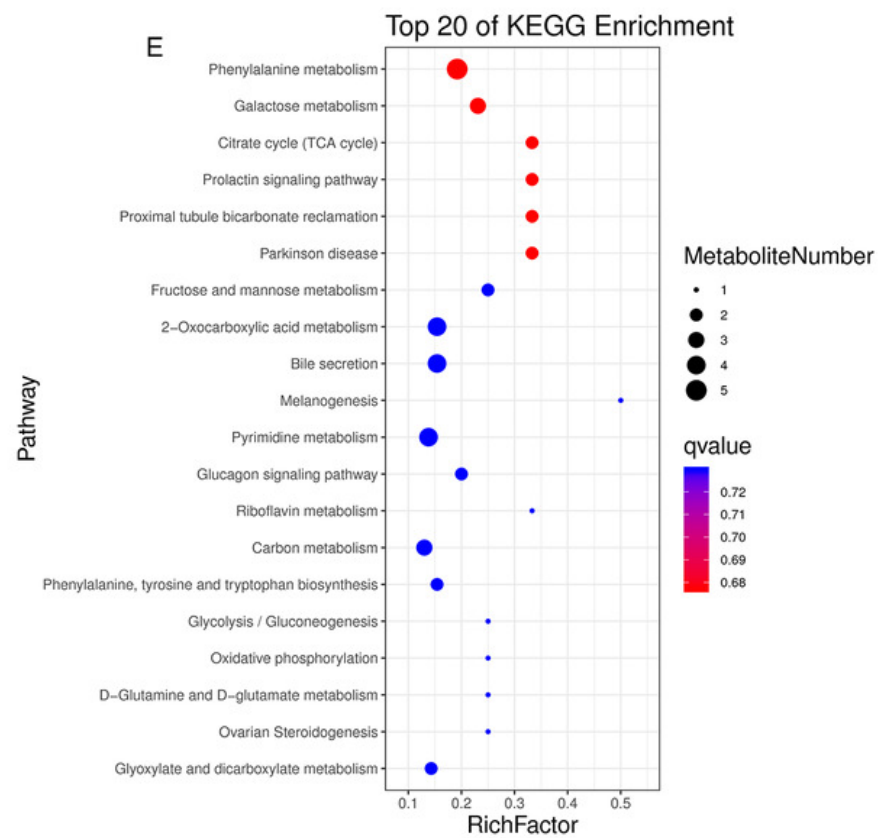


Figure 4

OPLS-DA score plot of of AKI-B vs. AKI-A and AKI-B vs. NAKI-B

(A, B) OPLS-DA score plot of AKI-B vs AKI-A, eighteen and twenty-three up- and downregulated metabolites, respectively, in AKI-B group compared with the AKI-A group; (C, D) OPLS-DA score plot of AKI-B vs NAKI-B, twenty-three and twelve up- and down-regulated metabolites, respectively, in AKI-B group compared with the NAKI-B group. Abbreviations: OPLS-DA, orthogonal projection to latent structures- discriminant analysis; A, preoperative sample; $B$, postoperative sample. 
A

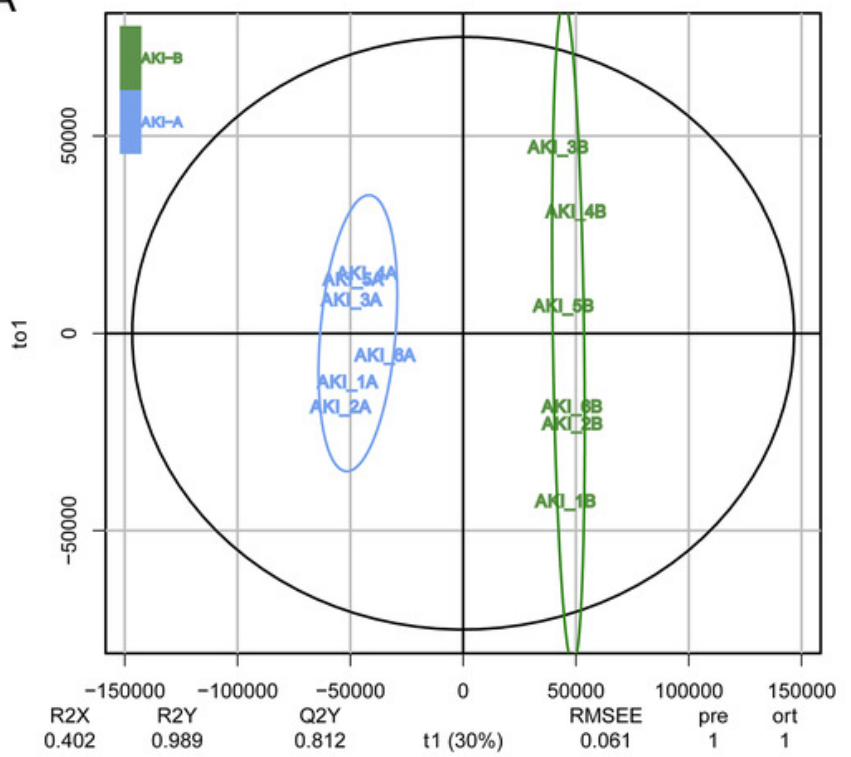

Scores (OPLS-DA)

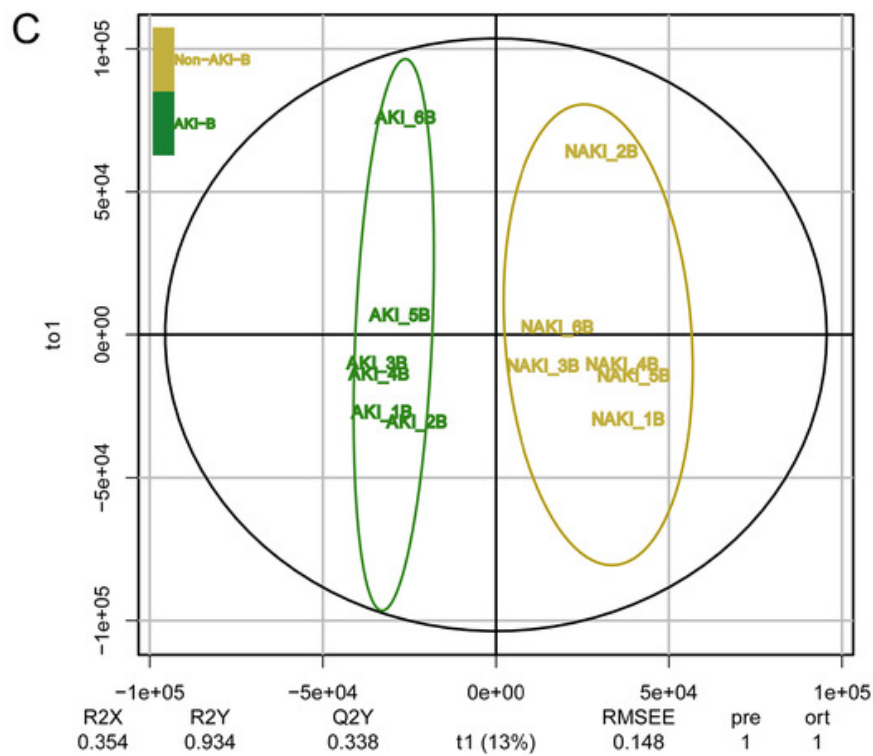

B

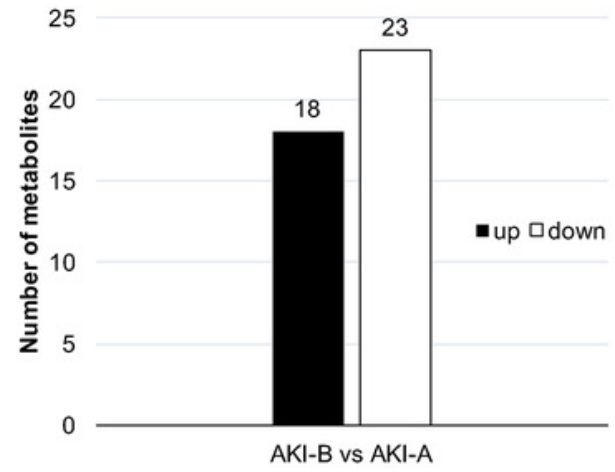

D

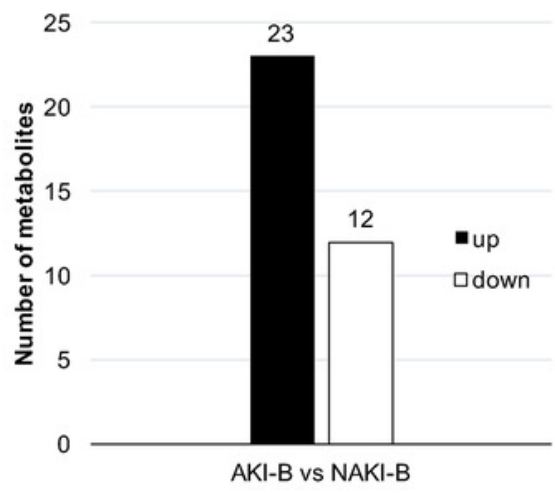




\section{Figure 5}

Venn analysis identified metabolites associated with postoperative AKI in DM

(A) Venn diagram of the up-regulated overlapping metabolites from three pairs data (B vs. A, AKI-B vs. AKI-A and AKI-B vs. NAKI-B); (B-F) The relative intensities of the 5 up-regulated overlapping metabolites before and after CABG; (G) Venn diagram of the down-regulated overlapping metabolites from three pairs data (B vs. A, AKI-B vs. AKI-A and AKI-B vs. NAKI-B); $(\mathrm{H}-\mathrm{K})$ The relative intensities of the 4 down-regulated overlapping metabolites before and after CABG. Abbreviations: CABG, coronary artery bypass grafting; A, preoperative sample; $B$, postoperative sample. 
A

Up-regulated metabolites

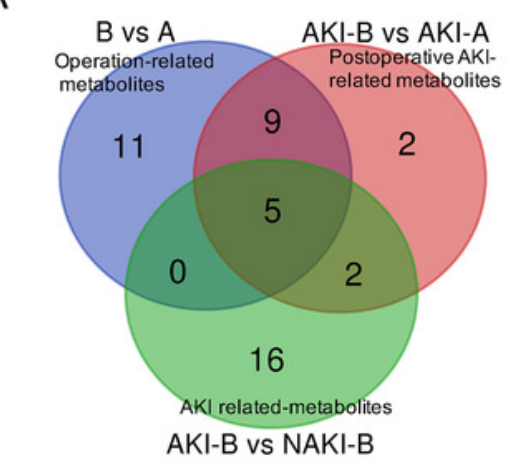

D

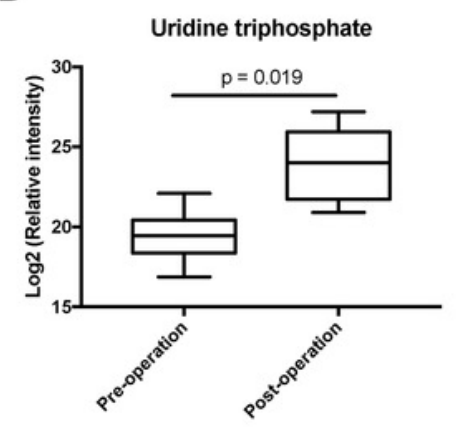

G

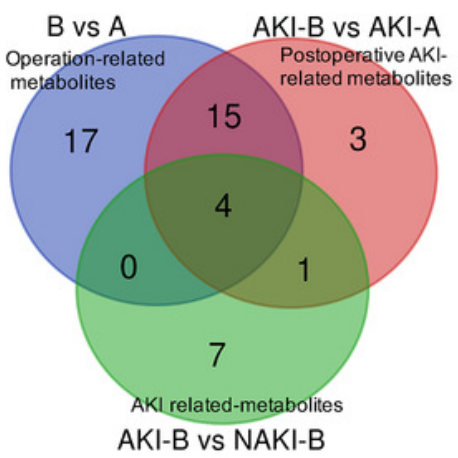

B

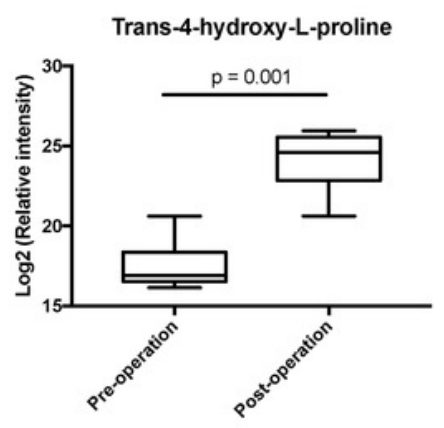

E

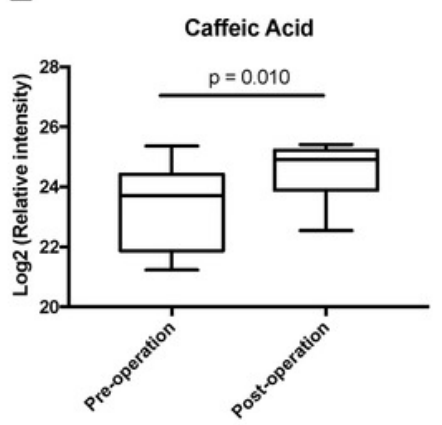

$\mathrm{H}$
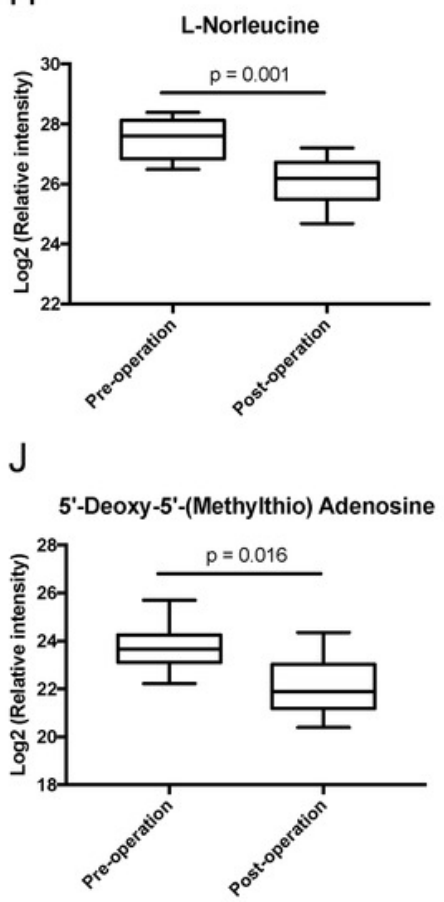

C

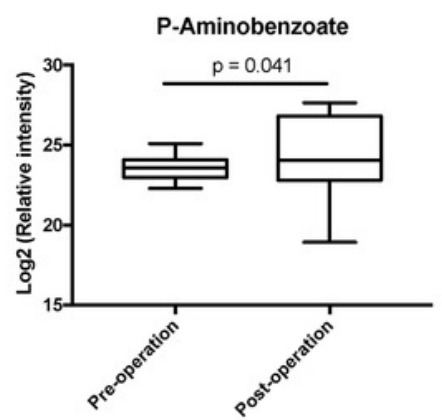

F
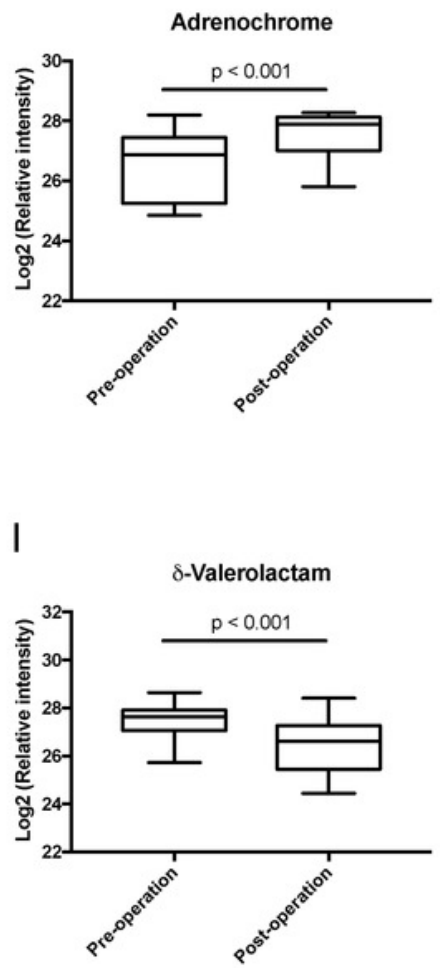

K

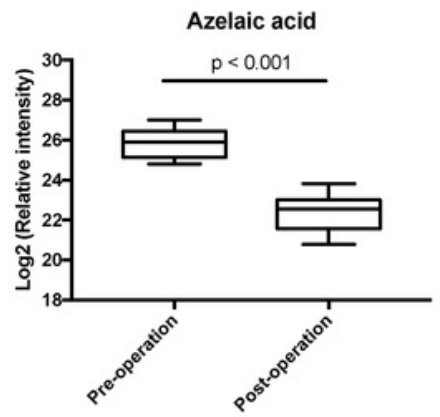




\section{Figure 6}

Urine metabolomic abundance in validation cohort were measured by targeted metabolomics analysis

(A) The absolute concentration levels of trans-4-hydroxy-L-proline in NAKI, AKI, DN-micro and DN-macro groups; (B-C) ROC curves of trans-4-hydroxy-L-proline for distinguishing AKI from DN-micro and DN-macro patients; The sensitivity (true positive rate) is set as the ordinate, and 1-specificity (false positive rate) is set as the abscissa. (D) The absolute concentration levels of azelaic acid in NAKI, AKI, DN-micro and DN-macro groups. (E) Correlation of azelaic acid with eGFR in cohort patients. Pearson correlation coefficients $(R)$ is provided in; (F-G) ROC curves of azelaic acid for distinguishing NAKI from AKI patients, DN-micro from DNmacro patients. $p<0.05$ was considered significant. ${ }^{*}, p<0.05$ vs NAKI group; \#, $p<0.05$ vs AKI group; $\&, p<0.05$ vs DN-micro group. Abbreviation: ROC, receiver operating characteristic curves.

A

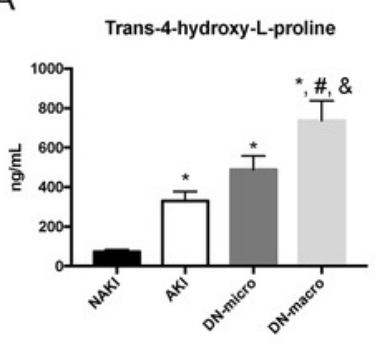

B

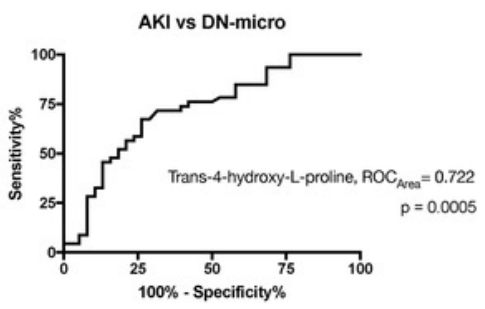

C

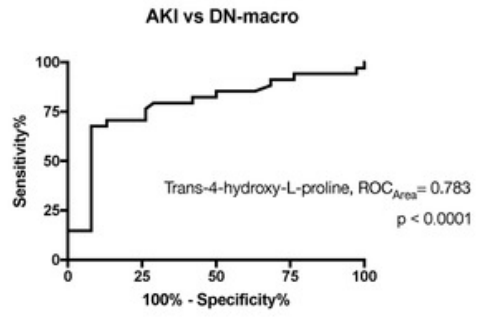

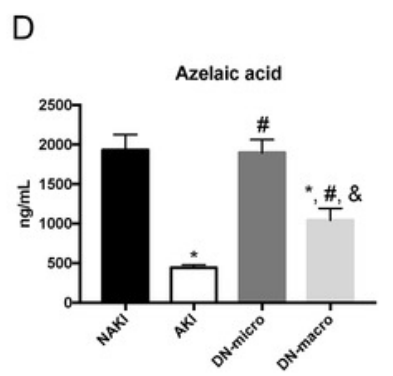

$E$

$\mathrm{F}$

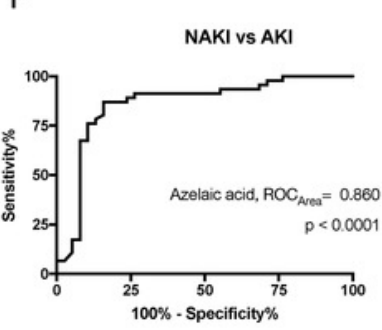

G

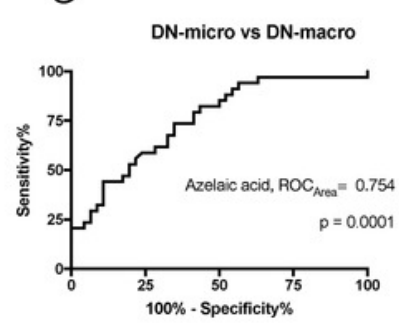

